\title{
Towards Memory Supporting Personal Information Management Tools
}

\author{
David Elsweiler, lan Ruthven and Christopher Jones \\ \{david.elsweiler, ian.ruthven, cjones\}@cis.strath.ac.uk \\ Department of Computer and Information Sciences, University of Strathclyde \\ Livingstone Tower, 26 Richmond Street, Glasgow, G1 1XH
}

\begin{abstract}
In this article we discuss re-retrieving personal information objects and relate the task to recovering from lapse(s) in memory. We propose that fundamentally it is lapses in memory that impede users from successfully re-finding the information they need. Our hypothesis is that by learning more about memory lapses in non-computing contexts and how people cope and recover from these lapses, we can better inform the design of PIM tools and improve the user's ability to re-access and re-use objects. We describe a diary study that investigates the everyday memory problems of 25 people from a wide range of backgrounds. Based on the findings, we present a series of principles that we hypothesize will improve the design of personal information management tools. This hypothesis is validated by an evaluation of a tool for managing personal photographs, which was designed with respect to our findings. The evaluation suggests that users' performance when re-finding objects can be improved by building personal information management tools to support characteristics of human memory.

\section{Introduction}

In our daily lives we constantly interact with a wide range of electronically stored information objects; email messages, web pages, digital images, video samples, etc. The sheer quantity of the information we create and use combined with limitations of human memory means that we cannot rely solely on our memories to recollect precisely what information we have seen, where we may have stored an object or how we can find it again. Consequently, we are forced to rely on tools to support our access and management of digital information. These tools are either dedicated to searching our personal information stores, such as Stuff-I've-Seen [Dumais et al. 2003], or are tools which allow us to manage information objects, e.g. folders in email applications. Information management tools are intended to help people find previously stored information by allowing the user to organise their information
\end{abstract}


objects. However, both the searching and managing approaches place the load for successful recovery of information on the user's memory.

To conduct a successful search on a query-based system such as Google desktop, for example, a user must remember sufficient details about the information they want to retrieve in order to form a query. However, psychological research indicates that people are not good at remembering precise details. Instead what tends to be remembered are high-level meanings or gists [Sachs 1967; Clark and Clark 1977; Rubin 1977]. This suggests that people would not be adept at remembering terms in a document, the subject of an email etc. - the kind of recollections required to construct queries.

The major alternatives to query-based systems are browse-based systems in which a user looks through information objects in order to find the objects they want. Browsing systems either show users all the objects available, limiting the approach to relatively small data sets, or force a classification on the objects such as colour distribution for images [Heesch \& Rüger, 2004], concepts for documents [Yang 1994], etc.

Similarly, information management tools force a classification on users, either by automatically classifying objects, as in text categorisation systems [Hayes et al., 1990], or forcing users to classify objects, usually in some form of hierarchical system [Malone 1983]. For example, photographs and music are generally organised in albums and possibly further sub-categorised by artist, date, genre etc. Operating systems manage applications and files in a hierarchical system of folders, email tools provide facilities to group messages hierarchically, and standard web page book-marking features are hierarchical.

Despite their popularity, hierarchical systems have been shown to have problems. Malone's study of natural office behaviour demonstrated that they are cognitively challenging and that users are reluctant to use them either because they cannot decide how to categorise an item, or because they are not confident in their ability to retrieve a categorised item at a later date [Malone 1983]. Similar behaviour has been observed with digital documents [Boardman \& Sasse 2003] and email messages [Whittaker \& Sidner 1996].

The limitations of existing Personal Information Management (PIM) tools and the fact that the quantities of information people are required to process are likely to continue to grow combine to motivate our work. In particular, we are interested in the role that human memory plays in the management of personal information. In PIM people try to obtain information based on the features of an object that they can recall. Therefore, the information that people forget is the barrier to successful 
retrieval - if they could remember everything that they once knew about an object then it would be simple to re-access it. To improve PIM systems we need to understand in more detail what people can remember, what strategies are successful for remembering and how we can design tools that better support personal information management.

The role memory plays in PIM is non-trivial and involves different types of memory. For example, when re-retrieving an object from our personal stores our strategy may be based on the recollection of a property that object has (semantic memory), a previous experience with the object (autobiographical memory), a temporal reference to that object, such as when it was previously accessed, etc. Depending on the context of the search it may be easier for the searcher to utilise some types of memory over others, e.g. in email retrieval, it may be easier to remember who sent an email, when it was sent or what it said depending on properties of the email and the search. Thus, supporting PIM should, we argue, allow for searchers to utilise different types of memory in retrieval. Further, it is lapses in memory, such as a failure to recall the specific location, property, or source of an object that prevents successful reretrieval in PIM. For example, in the period shortly after an information object has been stored or accessed it can be re-accessed with ease because the recollection of the object and its location is lucid. However, popular theories of memory emphasize the transient nature of human memory; recollection diminishes over time [decay theory e.g. Rubin \& Wenzel 1996] and focusing on other tasks and interaction with other objects can also degrade the recollection [interference theory e.g. Bower et al. 1994].

We hypothesize that in order to ascertain which types of tool will be effective, and how existing tools can be changed to enhance rather than restrict human recall, it will be useful to investigate memory lapses in other contexts: what do people forget, why do they forget and what automated support might make the process of remembering easier? Further, as we show in section 4, there are similarities between memory lapses that people suffer from and learn to deal with effectively in everyday life and those that hinder PIM. Therefore, can lessons be learned from everyday behaviour with respect to improving PIM practises and tools? These are questions we address in this work.

This article is divided into two main parts. In the first part, we report on a diary study that evaluates the variety, frequency and severity of everyday memory lapses. The study also explores the types of tasks that cause memory failure (or the memory failure to be reported), as well as the methods employed to recover from lapses. By comparing and contrasting the recorded memory problems and compensatory strategies with those that hinder PIM, we demonstrate restrictive aspects of existing PIM 
systems and their interface designs. We discuss the possible implications this work has for the design of PIM tools and illustrate them in the context of a tool for the management of personal photographs. In the second part of the article we deal with the main aim of this work - to determine if taking memory into account in the design of PIM systems is advantageous. To this end, we perform a pilot evaluation comparing the performance of our tool with a traditional browse-based interface.

The remainder of this article is structured as follows: Section 2 describes the background literature; section 3 details the research methodology used to examine everyday memory lapses; section 4 provides the results of the study, the implications of which are discussed in section 5, outlined as a series of design principles. Section 6 presents a tool for managing personal photographs, which embodies the principles established from our findings. Section 7 presents an evaluation of the tool. Finally, our conclusions are presented in section 8 set against the context of future work.

\section{Related Work}

This section describes the background literature for the primary themes of this article. Section 2.1 describes previous studies that have also taken a psychological approach to investigating PIM behaviour. Section 2.2 presents previous work that that relates memory lapses and PIM. Section 2.3 describes knowledge of everyday memory problems, while section 2.4 details methods for studying everyday memory problems.

\subsection{Personal Information Management Behaviour}

Several studies have been performed that have investigated personal information management behaviour in natural settings. These studies had the goal of uncovering the strategies people employ when storing and retrieving information, the reasons why they choose to use these strategies and the problems they have when doing so.

It has been observed, for example, that documents are often placed in piles rather than being filed in a more appropriate location [Malone 1983]. A number of explanations have been offered for this. Firstly, it is the result of people having multiple and conflicting uses for their document collections. Barreau and Nardi [1995] discovered that people use collections both for preserving information that they may need at a later time and for reminding themselves that tasks have still to be completed. Piles are common because, to a certain extent, they achieve both of these goals. When the number of documents in the collection remains small it can be easy to re-find sought after documents. Further, piles represent a kind of short-term memory; a buffer which retains tasks that must be performed [Jones 
et al. 2002]. This is useful because when documents are filed in folders you have an "out of sight, out of mind problem" [Bruce et al. 2004]. It is only when the number of files / piles scales beyond a certain threshold that the disadvantages of employing a piling strategy become apparent. In this situation different groups of people react in different ways. "Frequent filers" file documents as they use them and never let piles become large enough to cause trouble, "spring cleaners" respond to over-sized piles by archiving certain files into longer-term storage, whereas "no filers" make no efforts to manage the piles and struggle to work productively [Whittaker \& Sidner 1996].

The use of piling as an information strategy demonstrates that the function of the information space plays a role in determining how people manage that space. Kwasnik [1989a] also observed that the function or use of a document or specific elements within a document influences the way that people will store or file that document. For example, resources for teaching may be stored together. Bruce [2005] argues that it is the user's predicted need for information, i.e. their estimation of the value that the information may hold for them in the future as well as the reason for that importance, that have the greatest influence on the way they store it. Again, there is a problem with this because if people inaccurately predict future needs the information becomes difficult to retrieve when they require it for a purpose unrelated to its filed location.

Other researchers have observed that people use different management strategies depending on the format of the information [Kwasnik 1989b], their role within a company [Jones et al. 2002], and their relationship with the information [Jones et al., 2002]. In their studies of keeping information found on the web, Jones and others [2002, 2003] and Bruce and others [2004] observed many strategies for retaining information from web pages. They discovered that people, for example, use bookmarks, email themselves URLs, print out entire web pages, cut and paste useful information into other kinds of document. If the above studies are correct, then the exact method of retention will depend on a complicated array of factors. The lack of a well defined or easily predictable storage strategy places further burden on the memory when re-retrieving documents because to retrieve the document the user must remember contextual facts such as the tool used to retrieve it, the task they were undertaking at the time, their location etc. to determine where they would have stored the information.

Capra and Perez-Quinones [2003] noticed that when re-retrieving information objects users take a two-stage iterative approach. The first stage identifies an appropriate information source, while the second focuses on narrowing towards specific information from within that source. Their findings align with those of Teevan and her colleagues [2004] who discovered similarities between the way people re- 
find information and behaviour associated with orienteering. Orienteering as they describe it "involves using contextual information to narrow in on the actual information target, often in a series of steps". Teevan and her colleagues also observed a second approach to re-finding, which they refer to as teleporting; where users attempt "to take themselves directly to the information they are looking for" [Teevan et al. 2004]. An example of teleporting would be using a remembered URL to directly access a web page or using extensive, detailed search queries to locate a web page with one attempt.

Thus, a lot of data has been assembled from previous research. The findings show significant overlap between behaviour in physical and digital environments and provide an insight into the psychological reasoning behind this behaviour.

\subsection{PIM and Memory Problems}

Other researchers have related PIM to memory and weaknesses in memory. For example, Lansdale [1988] described office organisational problems as problems of psychology; more specifically problems of categorisation, recognition and recollection; Case [1991] proposed that memory and metaphor impact the way historians manage their resources; and Carroll [1982] demonstrated that simple eight character filenames can trigger a detailed recollection of a file's content. Capra and Perez-Quinonez [2005] suggested that the task of re-finding information is different to that of information discovery and involves different cognitive processes: information re-finding involves processes of recollection and memory, while finding new information involves processes of recognition. Capra and Perez-Quinonez suggest that re-finding tools should therefore differ from traditional search tools to support features of memory.

Studies conducted as part of the Keeping Found Things Found project [Jones et al. 2002, 2003, 2005; Bruce et al. 2004] found that memory problems hindered participants' ability to re-find all of their useful documents. Participants were generally good at getting back to a desired information item (e.g., edocument, email message or web page) if they remembered to look for it. However, participants complained that they often forgot to look for an information item until the period of its usefulness had passed [Jones et al. 2005]. Czerwinski and others [2002] investigated peoples' memory for everyday computing and found that users forgot a significant number of computing events that they had deemed important for remembering later during the original recording sessions. In addition, Czerwinski and her colleagues found that users initially had an excellent memory for the temporal order of events, but this knowledge decayed significantly over time. Further, several scholars have reported on how interruptions can upset users' primary tasks both in the context of PIM and general computer use 
[Renaud 2000; Czerwinski et al. 1991]. When this happens users struggle to remember the context of previous tasks in order to continue with this task [Czerwinski et al. 1991].

Despite recognising that memory has a function in personal information management behaviour and that memory lapses hinder PIM practises, little has been done to firmly establish what the function of memory is and how we could use this knowledge to improve the tools at our disposal.

In this article we build on the premise that memory lapses hinder PIM and examine other everyday memory problems to learn about possible ways to solve PIM lapses. We propose that the similarities between general lapses of memory and the memory lapses associated with PIM could be exploited and the strategies people employ to overcome general lapses incorporated in PIM tools.

\subsection{Everyday Memory Problems}

Everyday memory problems i.e. memory lapses that can occur as part of going about daily life activities, can be diverse, falling into the three categories: retrospective memory problems, prospective memory problems, and action slips [Eldridge et al. 1992, Terry 1988].

- Retrospective memory problems are concerned with forgetting details of past events or information acquired in the past. Examples include: forgetting the name of a person, place or object, forgetting the location of an item, or forgetting a detail about a past event, etc.

- Prospective memory problems are failures to remember a future task or event. Included in this category are problems such as: forgetting to go to the bank, forgetting about an appointment, etc.

- Action slips are concerned with very short-term memory failures which cause problems for the actions currently being carried out e.g. forgetting what one is doing following an interruption, forgetting why one went upstairs, or losing one's train of thought etc.

Investigations have drawn links between action slips and prospective memory problems, concluding that interruptions and task switching lead to failures in prospective memory [Czerwinski \& Horvitz 2002]. Little is known, however, about the effects that retrospective memory failures have or whether we have similar coping mechanisms for these sorts of difficulties. Are certain tasks responsible for memory problems and do our performances on these tasks suffer as a result? Memory lapses related to PIM or experienced while performing PIM tasks may also provoke task deterioration. Similarities between everyday lapses and those associated with PIM could mean that a study of memory lapses in a natural, non-computing environment and a comparison of these tasks with PIM memory lapses, may 
offer explanations for the deficiencies of existing computer-based PIM tools and attain clues about the best way to improve them.

\subsection{Methods of Studying Everyday Memory}

Studies of everyday memory phenomena can be placed in four categories: (1) experimental simulation and laboratory tests [Ebbinghaus 1885], (2) direct observation [Neisser 1982], (3) inventories and questionnaire based investigations [Hermann \& Neisser 1978], and (4) diary studies [Crovitz \& Daniel 1984].

Laboratory based experiments have provided useful information about the capabilities of human memory, its capacity and time periods of data retention etc. Unfortunately it is difficult to map the demands placed on memory in everyday situations onto standard laboratory experiments [Sunderland et al. 1983]. Consequently, studies exploring everyday memory are generally conducted in natural environments. Investigation through direct observation is a costly and time consuming practise with long research periods needed to establish natural conditions. Herrmann [1982] cited the difficulty in conducting ecologically sound research as the main reason for the neglected study of everyday memory.

Memory questionnaires have been suggested as a method of simplifying ecological investigation; see [Herrmann 1982] for a review. These establish participants' subjective perception of their memory performance by asking about "forgetting, remembering, memory change, memory use, and attitudes about memory" [Herrmann 1982]. Memory questionnaires have discovered frequency information regarding a range of known memory problems, although uncertainty exists over the reliability of the results, determined by participants' ability to evaluate their own memory performance [Sunderland et al. $1983]$.

An alternative methodology for establishing the performance weaknesses of everyday memory is the diary study. Rieman [1993] presents the diary study as a "middle-ground solution to the opposing limitations of laboratory and field studies" [Palen \& Salzman 2002]. Two diary studies of particular note [Eldridge et al. 1992, Terry 1988] have provided classification schemes characterising memory problems that humans can expect to encounter during their daily lives. Their use of homogenous population groups, however, restricts the usefulness of the work; participants in these studies were either researchers with similar educational and vocational experience [Eldridge et al. 1992] or psychology students from one particular class who also have similar backgrounds [Terry 1988]. Literature also exists on specific problem areas of memory, such as Loftus' work [1979, 1988 etc.] on eyewitness testimony and memory abilities for particular types of information [Cohen 2004]. 
The aforementioned studies focussed on specific groups or tasks. We, instead, seek to explore the distribution of memory failures in an everyday setting using a broad population range. This is because, despite a number of previous investigations, our knowledge of everyday memory problems remains limited. Further, the management of personal information objects and the problem of information overload are not restricted to specific groups of people. The popularity of digital devices such as cameras and music players, as well as new information channels like the Internet, email and digital television has made the problem universal. Thus, to build on previous everyday memory failure research, we performed a three-part investigation of the memory problems of 25 people with varying ages and backgrounds. Our investigation mainly centred on a week-long diary study. The intention was to uncover memory weaknesses from which people suffer and the behavioural changes and strategies employed to overcome and compensate for these. The ultimate aim was to increase our understanding of the attributes of memory with respect to developing improved PIM tools.

In the following section our methodology is outlined and justified with respect to previous studies of everyday memory problems and other studies that employed similar diary methods.

\section{Method}

Diary Studies offer the ability to capture factual data, in a natural setting, without the distracting influence of an observer. Limitations of the technique include difficulties in maintaining participant dedication levels and convincing participants that seemingly mundane information is useful and should be reported [Palen \& Salzman 2002]. The effects of the negatives can be limited, however, with careful design and good implementation. For instance, to help participants understand what kind of information should be recorded example situations can be provided and corresponding sample diary entries explained. Further, to maintain levels of participation the diary can be designed in a way that it requires minimal effort to carry and complete.

To complement the diary study a pre-study questionnaire and mid / post-study interviews were employed. The questionnaire was used to establish participant profiles, determining the amount of information they are exposed to, how organised they are and how they process such information, as well as discovering whether techniques or devices are commonly used to support memory. Participants were asked to rate themselves in terms of how organised they are generally and with respect to organising their computer files, personal music, photographs and web-bookmarks. The questionnaire also had a section to establish how participants rated their own memories. The use of frequency scaled questions, allowed the regularity of example lapse types to be established. This section also reinforced to 
participants that a wide variety of everyday memory lapses exist and that these should be recorded in their memory diaries. The questionnaire layout is shown in [APPENDIX A]. In addition to a final post study interview, the authors had regular conversations with participants as the diary study progressed. Despite their informal nature, both varieties of interview provided useful information, such as the attitudes of participants towards the study and their thoughts and opinions at various time points. Additionally, elaborative information was unearthed regarding diary entries of particular interest, including those that required several attempts to solve or remained unsolved.

At the outset, 30 individuals participated in the study. However, other commitments and demands the study placed on participants' time, meant that only 25 (15 male / 10 female) completed the process. The participants were of various ages; the youngest was 19 , the eldest 83.12 of the participants were aged 19-29, 7 were aged 30-49, 2 were aged 50-69 and 4 were aged over 69. Participants had diverse backgrounds, differing employment and educational experiences, as well as organisational habits.

The participants were recruited in various ways in an effort to include a wide variety of ages and backgrounds. A large number of the participants were university employees from within the Department of Computer and Information Sciences. These included clerical (3) and cleaning staff (2), academic (2) and research staff (2), as well as research students (3). To attract older participants (4), we advertised at a local senior citizens' swimming club. The remainder of the population consisted of university graduates now employed as software engineers (4) and those who responded to advertisement in a local public house (5). The last group included police officers, money advisors and an engineer.

Participants were provided with a pocket-sized pre-printed diary and were asked to keep an accurate $\log$ of memory lapses, as they occurred during their daily lives, for a period of a week. Each page in the diary captured information about a single lapse and details of the recovery from that lapse [Figure 1]. Details recorded included the time and date the problem occurred, how frustrated the problem made the participant feel, the task or situation that participant was engaged in when the problem was realised, and whether or not the participant had a solution in mind to recover from the problem. Regarding the solution, diary entries recorded the method of recovery or solution, the time taken to recover from or solve the problem, the effort taken to recover or solve, and the participant's satisfaction with the end solution. There was also a box to record the number of attempts required to solve the problem. If the number of recorded attempts was high, this may have demonstrated a high level of importance attached to the solution, as well as indicating failures in the retrieval process. The diary was designed to maximise the amount of data recorded, while minimising the effort required by participants. For 
example, scaled values were provided for measures of frustration, effort and satisfaction because we needed more than binary judgements e.g. "this problem frustrated me", but realised that participants would be unwilling or unable to describe the reasons for their frustration. Further, we used the diary design as a means to control the amount of data that participants provided. For example, the amount of space given to describe the context of a memory problem guided participants towards providing a consistent amount of detail in their descriptions. If we had merely asked participants to note down lapses on paper as previous studies did there would have been no control over this.

To evaluate and refine the methodology, as well as finalise an effective diary design, a week long pilot study featuring 6 subjects was conducted. Pilot subjects trialled two diary designs and their feedback was used to establish the final version [Figure 1]. Neither of the original pilot diary designs was used in the final study. Instead, we used the elements from each that participants preferred and the explanations given for the preferences to construct a new final diary design. One example of design change influenced by the pilot was the way in which participants recorded the time taken to solve a lapse. In one pilot diary participants were asked to record the date and time the lapse was solved, in the other they were given a check box scale to indicate, roughly, how much time had elapsed since the lapse occurred. Participants with the first diary did not like recording the time and date; finding it an arduous task. Participants with the second diary were happy with the scale. However, we had chosen the values on the scale poorly. Consequently, we devised a scale better suited to the time periods taken to recover from lapses in the pilot.

The semi-structured diary format allowed the capture of, not only descriptions of each lapse, but also contextual information about the circumstances in which the lapse occurred and later resolved. The methodologies employed in previous studies of everyday memory lapses could not provide this information because their participants were not given any strict template conveying what to record about the lapses nor given any particular medium to record the lapses. 


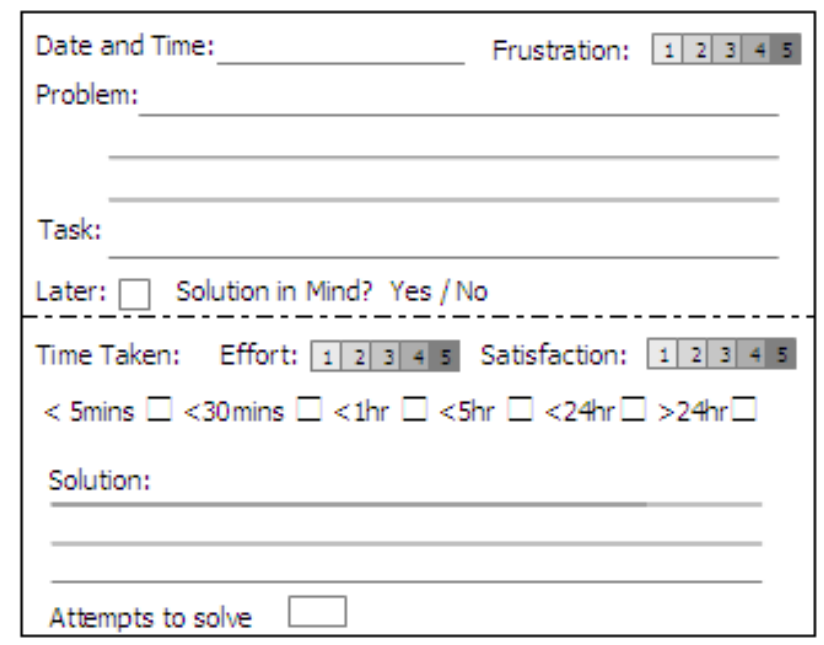

Figure 1: The Layout of the Diary Forms

\section{Results}

Several analyses were performed on the diary data. The following sections present the findings.

\subsection{Nature and Density of Memory Lapses}

During the diary study 261 lapses were recorded. 10 of the lapses were related to the diary itself. Examples of diary caused lapses included forgetting to bring the diary to work or forgetting the date when completing a diary entry. Such lapses were ignored from the findings. The lapse rate of this study (1.43 lapses per participant per day) is notably higher than previous studies: Terry (0.36), Eldridge and others (0.06). We believe this reflects an improved accuracy, resulting from an enhanced methodology; in contrast to previous studies we provided a means to capture data - a pocket sized diary - which participants were instructed to keep on their person allowing them to record lapses as they occurred. As previous studies did not provide a diary, this meant that if writing materials were not available when a lapse occurred it is unlikely that the lapse would have been recorded. Even though we found a higher lapse frequency than previous studies, the impression given by diarists is that the true frequency is much higher still. A number of participants stated that their diaries under represented the true frequency of the lapses. Two stated jokingly that "If I had noted everything I had forgotten, I wouldn't have had time to do anything else". Additionally, a number of entries reveal repeated lapses, for example, "Regularly misplace spectacles - often left home without them". Such lapses could only be treated statistically as a single problem. The frequency of recorded lapses and the comments given by participants emphasize the regularity with which memory lapses occur. 
Lapses recorded were categorised into a taxonomic scheme similar to that devised by Eldridge and others [1992], with lapses being classified into the same three main categories. However, within these main classifications, lapses were structured slightly differently. One new category of memory problem was created to evaluate retrospective information-based lapses. These include learning-based lapses. For example, forgetting where information was obtained or stored at some point in the past, losing details of processed information, such as facts from a viewed television programme or a read book etc. In previous taxonomies many of these lapses would have been categorised as forgetting details of a past experience. We feel it is more important, with respect to the goal of developing improved PIM tools, to highlight the participants' requirement to re-retrieve information that has already been in their possession. Thus, the classification scheme was created from the data we collected. The three primary categories remained as in Eldridge. However, the new inner categories were devised to better accommodate our data and research aims. The full classification scheme is detailed in [Table 1].

The majority of recorded lapses (51\%) were retrospective. $68.8 \%$ of these were information based while $31.2 \%$ were experienced-based. $35 \%$ of all recorded lapses were to do with attempting to reretrieve a piece of previously possessed information. This uncovers a substantial inadequacy in the way we deal with information and a need to improve our existing strategies or find alternatives, in order to remedy the situation. Prospective lapses also represented a large percentage (38\%) of the recorded diary entries. Action-slips represented just over $10 \%$ of the recorded entries. The lapse frequencies recorded in our study are closer to those of Eldridge, than to Terry's findings. Eldridge found that just under half the problems collected were retrospective memory problems; just fewer than $30 \%$ were prospective memory problems, while action slips accounted for just over $20 \%$ of the total. Terry, on the other hand, found that prospective memory problems occurred most frequently.

The frustration levels recorded were reasonably low $(\mathrm{avg} .=2.98)$. One diarist offered a possible explanation for this. He felt that frustration levels tended to increase the longer problems remained unresolved, but the frustration level was recorded when the problem was first noted. There was no great difference between the frustration levels recorded for the main category of lapses. Information-based lapses (avg. = 2.98), experience (avg. =3.3), prospective (avg. =2.96), action slips (avg. =2.92). When questioned on why some lapses were considered more frustrating than others, a common reply was that lapses were most frustrating when they knew the answer, but could not get access to it (tip of the tongue (TOT) sensations [Brown \& McNeil 1966]). Other situations described as particularly frustrating were memory lapses that caused embarrassment amongst peers and lapses where participants are sure that they had only recently known the solution. 
Table 1: Summary of Lapse Freq (excluding diary caused lapses)

\begin{tabular}{lccc} 
Memory Problems & Frequency & \% & Frustration \\
\hline Retrospective & 128 & 51.00 & 3.08 \\
\hline Information-based & 88 & 35.06 & 2.98 \\
\hline Forget a name & 30 & 11.95 & 3.00 \\
Forget a word & 5 & 1.99 & 3.60 \\
Information Processing detail & 11 & 4.38 & 3.67 \\
PC information & 5 & 1.99 & 3.20 \\
Forget fact & 33 & 13.15 & 2.61 \\
Forget item on list & 4 & 1.59 & 3.00 \\
\hline Experienced-based & 40 & 15.94 & 3.67 \\
\hline Misplace an item & 18 & 7.17 & 3.06 \\
Forget details of past experience & 18 & 7.17 & 2.75 \\
Forget a procedure & 4 & 1.59 & 2.96 \\
\hline Prospective & 97 & 38.65 & 4.00 \\
\hline Forget about an appointment & 1 & 0.40 & 2.80 \\
Forget to perform task & 69 & 27.49 & 4.00 \\
Forget to pass on message & 3 & 1.20 & 3.29 \\
Forget to bring item & 24 & 9.56 & 2.92 \\
\hline Action-Slip & 26 & 10.36 & 3.29 \\
\hline Forget to complete task after interruption & 7 & 2.79 & 4.00 \\
Miss out step in procedure & 1 & 0.40 & 3.50 \\
Forget the intention of an action & 4 & 1.59 & 2.50 \\
Lose train of thought & 14 & 5.58 & \\
\hline Total & 251 & & \\
\hline
\end{tabular}

It was observed that lapses, which occurred when using a computer system, seemed to mirror those found in non-computing contexts. This is evident across the taxonomy. For example, studies of office behaviour have shown that we misplace documents and have difficulties in retrieving specific information from documents just as we do in the physical world [Malone 1983, Whittaker 1996]. We forget to perform computer-based tasks in the same way as non-computer-based tasks [Dey \& Abowd 2000], and we are just as likely to lose our trail of thought and switch between tasks while operating a computer system. One explanation for this transfer of problems may be the way we design interfaces. We use design metaphors, such as the virtual desktop (files), photograph albums (photographs) and the clipboard to bring familiar physical world behaviour into a computing context. Without question design metaphors can make interaction with computer systems more intuitive; allowing new users to make inferences about software functionality from their existing knowledge bases and transfer skills to a new domain. The downside to this method of design, however, is that the problems we experience in the physical world are recreated when we operate computer systems. It would be interesting to investigate whether interfaces could be designed in such a way that the functionality could be intuitive without over-reliance on a central design metaphor. Using such a strategy may alleviate the transfer of memory weaknesses from physical to digital domains. We describe our initial efforts towards building and evaluation such an interface in sections 6 and 7. 


\subsection{Explanations for the Triggering or Recording of Memory Lapses}

The participants took advantage of a wide variety of different tools and techniques to support our memory [see section 4.3]. The tools, simple as they are, counter all three major memory lapse categories. Despite this support, memory lapses are still prevalent. Determining the cause of memory failures and the kind of situations in which they occur or are realised, may draw attention to behavioural aspects of our lapses.

Retrospective lapses occur when an individual realises a deficit in knowledge, but recognises that the information required has previously been in their possession. Prospective lapses are somewhat different and relate to absent-mindedness. Event-based prospective failures occur when a retrieval cue (event) fails to remind us to perform a task; either the event does not happen or is not strong enough to prompt us to perform the task. The successful completion of time-based tasks requires both the user to be aware of the current time at the moment the task should be completed, as well as the task to be resident in working memory at that time.

We analysed the diary entries to discover patterns in the recorded situational context that give clues to the reasons behind memory lapses or why the lapse was realised and recorded. The analyses were based on the details of the recorded lapse and the task in progress when the task was realised e.g. When the task entry mentioned "talking", "conversation" or "emailing" etc. these lapses were determined to be examples of communication caused lapses [see section 4.2.1]. If there was doubt about an entry this was clarified by asking the participant. The following sections attempt to characterise these recurring causal situations.

\subsubsection{Human - Human interaction.}

The diary study data suggests that socio-communicative processes can lead to memory lapses. $40.9 \%$ of information lapses and $24.7 \%$ of all recorded lapses were caused by communication. Sharing stories and reminiscing were common examples. For instance, while describing past events or telling stories participants were often made aware of the fact that they no longer had access to information. People can also serve as retrieval cues, bringing to light prospective memory failures. In our study people, actions performed by people and things said by people were often cited as the cause of a lapse. These can suddenly refocus the mind on tasks that should be or should have been completed. People are the cause of so many lapses because of their ability to remind us about other things. This could possibly be exploited in PIM interfaces. 


\subsubsection{Failed Multi-Tasking}

We found that attempting to perform multiple tasks simultaneously can be a further source of memory lapses. Other researchers have observed that time demands and lifestyle trends are forcing us to adapt our methods of working to incorporate multi-tasking [Rubinstein et al. 2001]. There are many consequences of multi-tasking behaviour and researchers in several fields including HCI [Czerwinski \& Horvitz 2002], Information Retrieval [Spink \& Park 2004] and Psychology [Rubinstein et al. 2001] are working to understand and support performing simultaneous activities and task-switching. Our results show that $14.34 \%$ of all recorded lapses were caused by multi-tasking. Nevertheless, very few retrospective lapses were caused in this way (6.8\% information, $10 \%$ experienced based). Despite the fact that previous studies have drawn a link between multi-tasking and prospective memory lapses [Czerwinski \& Horvitz 2002], our study revealed that only $15.5 \%$ of prospective lapses were caused by

performing multiple tasks. This is a relatively low amount when compared to action-slips, nearly half (42.3\%) of which were caused by attempting to multi-task. This is important with respect to our aim of supporting PIM because switching tasks while searching may detract attention from the search goal and lead to the loss of important contextual information and trails of thought. This suggests that PIM tools should provide features to counteract action-slips.

\subsubsection{Poor Encoding}

There are three stages associated with human memory [Eysenck 2001]. The first, encoding, is the process in which mental representations are created from external stimuli. As a result of encoding, some information is committed to memory (storage stage). The final stage is retrieval, where information is recaptured from memory. Poor encoding may seem an obvious explanation for a memory failure. The reasons behind poor encoding, however, are not so clear. Failure to predict a future information need and consequent failure to direct enough cognitive resources towards the encoding process is a principal cause of information-based lapses. Even when an information need is successfully forecast, the encoding process can fail to store the information as required. Inadequate encoding was clearly evident in the diary study findings, with participants often remembering a learning experience, but being unable to recall details of the learned material. This situation further explains the difficulties users have in creating queries to re-find personal information objects. For example, a user may remember the act of filing a particular information object, but forget exactly where the object was filed. This suggests that if PIM tools could support some means of enhancing encoding it may help re-finding. 
This section has described situations that lead to the occurrence of memory lapses. The following section outlines what we discovered about how participants overcome these problems and discusses what this means with respect to PIM.

\subsection{Overcoming Memory Lapses}

Participants in our study employed strategies to combat memory weaknesses. This was clearly evident in the findings of all three study components: the questionnaires, diaries and interviews. Further, from the pre-study questionnaire we discovered that every participant in the study utilised at least a one non-computing memory support technique. Many of the participants make use of tools such as calendars, timers and alarms. The use of electronic organisers / PDAs was less popular with only $19.2 \%$ making use of such devices. A surprisingly limited use of readily accessible computer-based organisational tools, such as web-favourites (bookmarks), email folders and music / image management software was also highlighted, although this has also been observed in other PIM studies [Jones \& Thomas 1997; Hightower et al. 1998]. Even when these tools are used, our results show that they are seldom used to their full potential. Bookmarks and emails were usually just filed in one large folder. When participants were questioned as to why they chose to adopt this behaviour, common responses were: "It takes too much effort to organise them", "I can't be bothered”, and "I usually just use a (web) search engine to re-find information". Such attitudes emphasize that users prefer to direct their efforts towards recovering from lapses rather than towards preventing them. This is another similarity between behaviour associated with recovering from general problems and observed PIM behaviour [Malone 1983]. Despite the preferred emphasis on recovery, the diary study and interviews also revealed a number of both preventative and recovery approaches.

\subsubsection{Preventative Strategies}

Preventative measures are mainly concerned with averting prospective memory failures and generally involve converting time-based cues (perform task $\mathrm{X}$ at time $\mathrm{Y}$ ) to event-based cues (after you perform task X, perform task Y). One common example was the use of objects left in specific places as task reminders. This was a strategy employed by several participants, but for different purposes. One participant leaves inanimate objects beside the front door to remind herself to take things with her when she leaves the house. This strategy fails when the cue (object) is not strongly enough connected with the item to be remembered and consequently, fails to re-trigger the task. Another participant uses a similar strategy to remind himself to record television programmes. By placing a video cassette on the floor, whenever he enters the living room he is reminded of the task. Once again the strategy fails if he cannot remember the details of the desired programme. 
Other preventative measures include writing information required or future tasks to be performed on one's hand, which is a simple and effective way to keep information for short time periods and the use of post-it notes etc. In the context of PIM the act of taking notes or filing documents can also be viewed as preventative strategies to counter information-based lapses and, as explained above, prospective lapses where by filing the user is attempting to remind themselves that a resource is available when they need it. These processes rely heavily on predicting future information needs $-\mathrm{a}$ task at which humans are usually not competent [Lansdale 1988].

\subsubsection{Recovery Strategies}

Recovery strategies are mainly associated with retrospective lapses and action slips, as when prospective lapses are realised it is usually too late to recover. Many different methods are employed to recover from memory lapses and these can vary strongly across situations and from person to person. Several mental strategies were revealed by the study. One participant described her approach to combating a tendency to forget names of acquaintances. "I work through names alphabetically and try to match them to faces in my head. I start at A: Andrew, Alasdair, Alex, Aaron.... etc. working through the alphabet until I match the face to the name ". Other participants described taking mental journeys to recapture information and recover misplaced items. Some described these journeys in a temporal fashion, relating information to events that they know happened at a particular time, while others used spatial information, such as particular locations to help retrieve lost information. One diarist described a mental journey he travelled to recall what he was working on at a particular point in time. "I'm not sure why, but I can relate conferences to work I was undertaking at that time. Maybe it is because the thoughts I generate while I'm there connect other peoples' work to my own at the time. So, when I wanted to remember what I was working on last year, I looked up what conferences had taken place at that time and I instantly starting making connections".

Another participant described spatially reliving events in his mind to locate a misplaced library book. The retrieval process is described graphically in Figure 2. The participant enhanced a physical search process by mentally reliving events and utilising various recollected visual and spatial contextual information to deduce likely locations. These "mental journey" examples show that similar techniques are used by different people, but the techniques are adapted to cater for individual needs and situations. Another recorded problem, investigated by interview, provides further examples of the processes undertaken during the recovery from a memory lapse. This lapse is shown in Figure 3. This example recording shows that memory lapses are not always independent. In this case, to avoid a prospective 
memory failure, i.e. successfully complete an assigned task, the participant must first recover from a retrospective, information-based lapse.

The participant had access to a number of pieces of partial information relating to the task. "I knew it was in one of two mini (car) magazines I had read recently and I knew it had something to do with nuts \& bolts". The diarist's first action was flick through the magazines, one after the other, looking for an effective prompt. Suddenly, he got the idea that the information he desired may be in an article by a particular writer. The contents page of one of the magazines was used to locate this article, which was subsequently scanned without successfully finding the desired information. Returning to the previous strategy of searching for cues, he arrived at a page where the layout seemed familiar. After reading that page the recovery process was complete and the participant had possession of a web address to lookup. Recovery from this lapse was far from a direct, predictable process. The participant had access to a number of information fragments; partial clues including fuzzy location and topic as well as visual information. How these clues were manipulated to retrieve the required information was completely dependent on the individual, his state of mind at the time, and the tools at his disposal. In this case, where the participant was retrieving information from a physical object (magazine), the tools were limited to the contents page and manual location of information and cues were restricted to information shown on the pages that were flicked through. However, in a digital information environment there is no reason why we should limit the user to such tools and cues. The findings here suggest that additional cues that are supplied to the user while they search may help them find the objects they are looking for. 


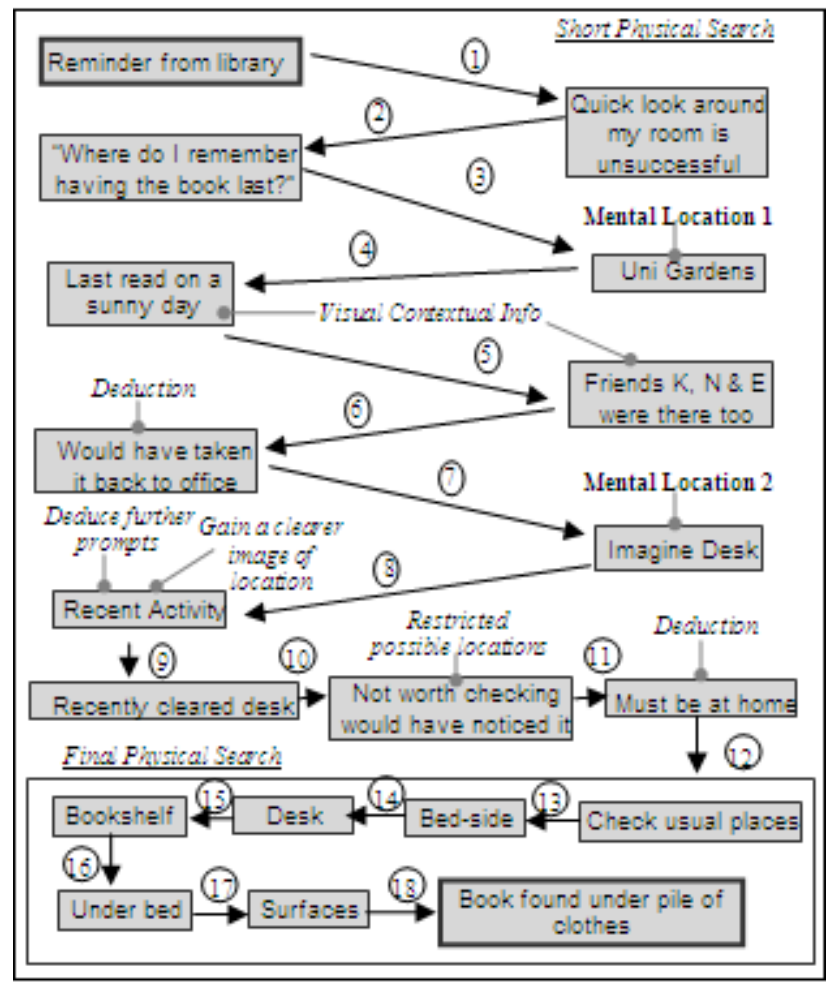

Figure 2: Visual representation of spatial mental journey

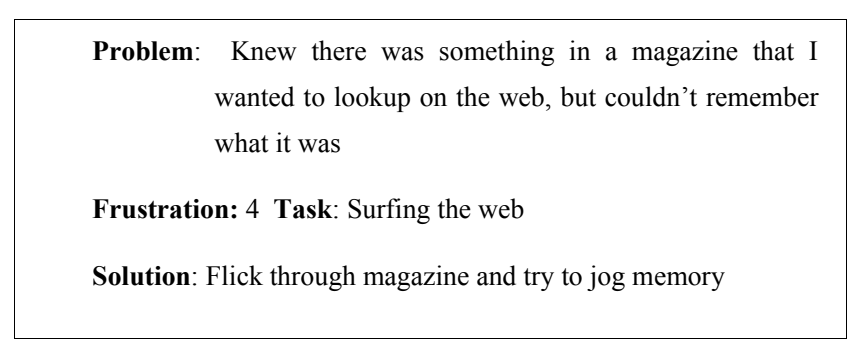

Figure 3: Example diary entry

\subsubsection{Sources for re-retrieving information}

The results reveal trends in the way that participants recovered from information based lapses. There were 89 information based lapses recorded during the diary study. 9 of these lapses went unsolved, 3 had no possible solution. Of the 77 lapses available, 71 gave enough details to discover the source of the solution. The results are shown in Table 2. A large percentage (23.9\%) of information problems were solved through interaction with friends and colleagues. From the interviews it was determined that people, if they are available, are usually the first choice source for re-finding information. The ease with 
which information to be attained in this way may explain this. The diary logs recorded people as the solution requiring least effort (1.90). Using the Internet to re-find information was also a popular way of solving information problems, accounting for $22.5 \%$ of solutions. This method also had a low effort score (2.06). The largest percentage of solutions was the grouped category for other external resource. However, this was a broad category incorporating a wide variety of solutions, including, looking up books, dictionaries, calendars etc. The effort levels associated with these recovery techniques was higher (2.53). The final category of solutions was solving without the use of external resources i.e. utilising mental techniques to jog one's memory. Solutions in this category also judged to require a large amount of effort (2.5).

Table 2: Solution methods for Information-based lapses

\begin{tabular}{ccccc} 
Memory Problems & Freq & \% & Effort & Satisfaction \\
\hline Information-based & 89 & - & - & - \\
\hline Unsolved & 9 & - & - & - \\
No Solution Available & 3 & - & - & 3.47 \\
\hline Information-based with solution details & 71 & 100 & 2.25 & 3.7 \\
\hline Solved by asking people & 17 & 23.9 & 1.82 & 3.67 \\
Solved by using the Internet & 16 & 22.5 & 2.06 & 3.17 \\
Solved by other external resource & 30 & 42.3 & 2.53 & 3.38 \\
Solved without external resource & 8 & 11.3 & 2.5 & \\
\hline
\end{tabular}

The findings are in-line with those of Hertzum and Pejtersen's [2000] for searching for information. They discovered that engineers search for documents to find people, search for people to get documents, and interact socially to get information without engaging in explicit searches. It is also in line with the work of Byström and Järvelin [1995] who showed that the more complex an information task appeared the more likely the task performer was to ask another human rather than an automatic information source.

Just as memory problems found in computing-context mirrored those in the physical world, prevention and recovery methods also transferred. We found that participants left files on the desktop and messages in their inboxs as reminders that they are active and to make them easy to find; an observation also made in PIM studies [Gwizdka 2000; Ducheneaut \& Bellotti 2001]. This behaviour was equivalent to behaviour with common physical objects, such as the video cassette example in section 4.3.1. The mental processes used to re-find information also equate, in both situations the user's behaviour depends on what user remembers, the facilities they have access to and their current state of mind. 


\section{Discussion \& implications}

Before discussing the implications, it is important to acknowledge the weaknesses of a diary study of this nature. The reliability of results will vary according to the motivation and performance of the participants, therefore we do not claim to have recorded every lapse the participants experienced. The methods employed only captured recognised memory lapses - participants may have never become aware of lapses and consequently they would have went unrecorded. We accept that memory failures will have been omitted from our results, but believe that the lapses that were recorded will have been those that particularly affected participant lives i.e. they recorded lapses because they had enough impact for the participant to recognise that they had occurred. Hence, the recorded lapses are the most important for our needs in this context. We also feel that we captured useful and accurate contextual information surrounding recorded lapses that enabled us to discuss participants' behaviour with respect to individual lapses.

In this paper we presented a study of the problems people have with memory and how people manage their memory problems. The aim behind this work is to understand how the design of PIM tools can support human cognitive abilities and to discover the type of features that are required.

The three varieties of memory lapse that were uncovered in our study can be related to memory lapses associated with PIM. As explained above, people re-find information objects to overcome retrospective information lapses and, in fact, overcome retrospective lapses in order to re-find information objects. People have been shown to pile documents as a way of creating reminders to overcome prospective lapses. Additionally, miss-filing documents can be the cause of prospective lapses. Even when documents are filed in a meaningful place, as noted by Jones and his colleagues [2005], people often forget to use documents when they need them because they forget that these documents are available i.e. there is no strong prospective cue to perform the reminding function. Furthermore, as previous research has indicated, action-slips are detrimental to PIM in that loss of concentration, due to a distraction or switch of task, can lead to search failure.

The findings provide a platform to discuss the merits and weaknesses of existing systems. Based on these we present a series of principles that may be evaluated, and if proven, may eventually form the basis of the framework for memory supporting PIM tool design. Our main conclusions are discussed below and implemented and evaluated in Sections 6 and 7.

We demonstrated that there is a wide range of forgetting behaviour and consequently a need for different types of tools to support memory failure. Our taxonomy is more detailed than those previously 
devised and uncovers a distinct need for information-oriented prostheses: tools that help users remember information. This finding re-enforces the motivation for our work. Re-retrieving information that we have already possessed was a common problem and one that is not effectively supported by current tools as existing support for re-retrieving information forces users to rely on a single recollection type to facilitate retrieval. Traditionally, interfaces rely on spatial recollection to access information objects. A good example of this are hierarchical systems [described in section 1], including the familiar desktop metaphor. Alternative metaphors have been proposed that utilise other types of recollection. The dimension of time has been advocated by several scholars to account for the fact that users are often able to distinguish temporal relationships between objects, events and dates [Freeman \& Gelernter 1996, Rekimoto 1999, Krishnan \& Jones 2005]. For example "I remember writing that report roughly around the same time as I attended the conference in Sweden". Information object properties have also been suggested as means to store and access personal information [Gifford et al. 1991, Dourish et al. 2000, Adar et al. 1999]; this represents a semantic dimension exploiting recollections such as "That paper is very long, but highly related to Jim's work'.

Although there is good evidence from cognitive psychological research endorsing the recollection modes utilised by the systems above, our diary study findings suggest that restricting interaction to any single dimension is counter productive, perhaps hindering re-access. The findings suggest that multiple modes of interaction should be incorporated into interfaces to support the fragments of recollection and individual mental journeys described by participants; supporting multiple forms of recollected context rather than relying on any single mode. The remainder of this article attempts to validate this hypothesis.

We demonstrated that an effective recovery strategy to cope with a memory failure is taking "mental journeys": rerunning previous interactions to jog the memory. The exact nature of these mental journeys are individual, e.g. some tasks were more suited to a visual, spatial journey, some to a temporal replaying, but the core finding is that recording previous contexts - the context in which objects were used can be the basis of tools for helping people remember. The findings suggest that if PIM interfaces could recreate some of this context when the user attempts to re-retrieve objects it could improve their performance.

We also demonstrated the overlapping nature of memory. Objects and other entries that would reside in semantic memory are automatically, possibly unconsciously, associated with tasks to be performed (prospective memory) and past experiences (autobiographical memory). These objects can serve as cues 
that bring the other thoughts and recollections to the forefront of the mind. We highlighted people as particularly effective recovery sources. The effectiveness of any cue, however, is a completely individual phenomenon. This was demonstrated in the example where a participant was trying to finding a particular magazine article. We suggest exploring how cues can be automatically generated and supplied to the user as they search to assist with re-finding information. We must discover what cues are effective and how best to distribute cues while a user interacts with a system with the intention of refinding information.

Finally, we demonstrated that one reason people have trouble remembering is that they do not always encode information effectively. We believe that lessons can be learned from the work of psychologists and mnemonists who have demonstrated that information can be encoded with greater efficiency through the use of mnemonic strategies: strategies for elaborately processing information. If computer interfaces could be designed to incorporate intuitive methods for managing information that implicitly incorporated such processing techniques, then the user's ability to retain information may be greatly improved. One way in which this could be achieved is by offering improved annotation facilities. The is discussed further in section 6.

To summarise, from our findings we have extracted the following design principles.

Interfaces should:

1. offer multi-modal access to information objects

2. promote retrieval journeys using small fragments of recollection

3. offer cues to help the user more as they search. Cues should be provided to remind users about objects they are looking for, the information space, objects that could be useful but the user has forgotten about, and tasks performed with the system.

4. recreate the contexts in which objects had previously been accessed, used or modified

5. offer annotation facilities that allow elaborative encoding in line with retrieval

This section has discussed our findings in relation to existing PIM systems and suggested ways in which these may be improved to better support human memory. Our study examined everyday memory lapses and the behaviour associated with preventing and recovering from lapses. We have drawn parallels with memory lapses hindering re-access to information and elicited hypotheses in the form of 
design principles for tools to support information management. The remainder of this article builds on this work by illustrating the findings in an application for the management of personal photographs. Photographs were chosen to demonstrate our theories because of current research interest in this area, the ease of finding evaluation participants with data collections, and our belief that participants would be more comfortable sharing their personal photographs than other types of personal data.

\section{Implementing our Findings in an Interface for Managing Personal Photographs}

Increased popularity of digital photography and resulting growth in the sizes of personal image collections has exposed the limitations of existing photograph management tools and motivated considerable research attention. Systems developed have generally followed the same trends as those for other objects; exploiting spatial, temporal, and semantic recollections. However, photographs have additional properties that can allow utilization of additional memory types such as visual recollection, strong autobiographic recollection etc. Further, photographs have characteristics that can be used for automatic classification, as well as inducing re-accesses e.g. colour histograms, time-stamps etc. Each of these has been considered in retrieval interfaces. Platt and others [2003], for example, examined the use of time and colour based clustering. Bederson [2001] exploited visual perception skills for recognition and recollection of personal images to create a zoomable photo access interface. Rodden and others [2001] also utilize a specific type of visual recollection in their evaluation of the usefulness of grouping images by colour similarity, while the ShoeBox interface [Mills et al. 2000] incorporates recollections of colour, texture, shape and sound.

Based on the findings of our diary study a new image browsing interface was created, which we refer to as "PhotoMemory". PhotoMemory was designed in an attempt to minimise the burden placed on the human memory when searching for personal photographs by providing support for the three primary lapse types uncovered in our study. The PhotoMemory interface was developed as a prototype to evaluate our findings [discussed in section 5] and to learn lessons to assist the design of future applications. The interface is shown in Figure 4.

In an attempt to incorporate elaborative encoding features as discussed in section 5 [design principle 5], the application provides facilities for the annotation of photographs. Descriptions can be attached to images, images can be placed in semantic groups and concepts can also be assigned to images. Photographs can be annotated as they are added to the system or annotated in parallel with the browsing process. The idea was to make the annotation of images as simple and effortless as possible, to 
encourage the elaborative encoding effects induced by annotation and to switch the emphasis from lapse recovery [as observed in section 4.3] to lapse prevention. The focus in this article, however, is not on annotation. Rather, we concentrate on the methods of interaction that the system promotes when reaccessing photographs. The design adds a UI layer on top of a faceted classification system [Yee et al. 2004], which offers a combination of searching and browsing.

\subsection{Retrieval Journeys}

PhotoMemory does not feature any of the design metaphors commonly associated with photo management applications, such as albums and collections. Instead, based on our findings, we attempted to design an intuitive, simple to use interface that facilitates the use of the user's existing memories and prompts enhanced recall.

The diary study revealed that a popular means of recovering from retrospective memory lapses is to take "retrieval journeys". These were either taken mentally, as in the library book lapse [figure 2], or physically, as in the magazine article lapse [figure 3]. Making journeys in this way helps people remember because it allows them to re-create previous facts or experiences in a controlled fashion piece by piece. We tried to incorporate this approach [design principle 2] into PhotoMemory by allowing users to interact with the system in the same way as they take these journeys. The premise is that when the user initiates a search they will remember something about the photograph(s) they wish to find. Previous work by the authors suggest that people tend remember contextual fragments about their photograph(s) [Elsweiler et al. 2005]. For example, they may remember that a certain individual was in the photo or the time or event at which the photograph was taken. Unlike many other photograph management tools, where these fragments would not be enough to find the photos they desire, the PhotoMemory interface allows any of their recollected fragments to be used to take small steps along their journey, moving them closer to the photographs they desire [design principle 1]. The idea is that while interacting with the system to take these steps the system can show information that helps the user remember more about what they are looking for, allowing them to move closer still. This pattern continues until they reach their destination i.e. find appropriate images. Or, put another way, recover from all of the memory lapses that impeded re-retrieval. We discuss how the system shows information to cue improved recollection in [Section 6.3].

Just as in the diary study, where participants tailored the basic mental journey strategy to their own needs and skills, the PhotoMemory interface allows the retrieval journey to be customised based on what they remember and which thoughts are triggered as they interact. The intended form of interaction is, 
therefore, similar to the behaviour observed by Teevan and others [2004] where users "narrow in" on their target information. However, our application supports this behaviour by attempting to induce new trains of thought and making journeys more fluid. We discuss this in greater detail below.

\subsection{Growing Paradigm}

To support the concept of retrieval journeys, in the PhotoMemory interface the user's full collection is visible on screen at all times. Photographs are never removed from the screen completely, only reduced in size. Users express what they remember about the photographs they want by applying filters that correspond to various types of recollection [discussed in section 6.3]. When filters are applied images that match the criteria grow while the remainder shrink, providing an interaction paradigm that should make it clear which photographs meet the filter criteria without eliminating any data from the user. The idea here is to maximise the potential for serendipitous acquisition of retrieval cues. If photos that did not match applied filters were removed we would have the "out of sight, out of mind" problem discussed above. However, because non-matching images are only reduced in size there is a chance that the user may come across non-matching images that reminds them of something that helps their search. The principle is the same as the preventative prospective-lapse strategies observed during the diary study. One further advantage of the growing paradigm is that because images are never removed the spatial relationships between images are retained i.e. images always have the same neighbouring images. This means that the visual and spatial contexts of previous interactions with photographs are maintained, which, in theory at least, should support the user's recollection [design principle 5].

\subsection{Offering Feedback to Users While They Search [Cueing Recollection]}

In an effort to build a fuller recollection of target photographs our interface provides users with stored contextual information based on their actions. For example, on mousing over a photo, a display is generated [Figure 4] containing a magnified version of the thumbnail, as well as its description and a list of other annotations including group classifications and time stamp information. Offering visual, contextual cues in this manner, in combination with drawing attention to growing images is hypothesized to subtly reacquaint users with previous experiences with images including any annotations that they have made in the past [design principle 3]. 


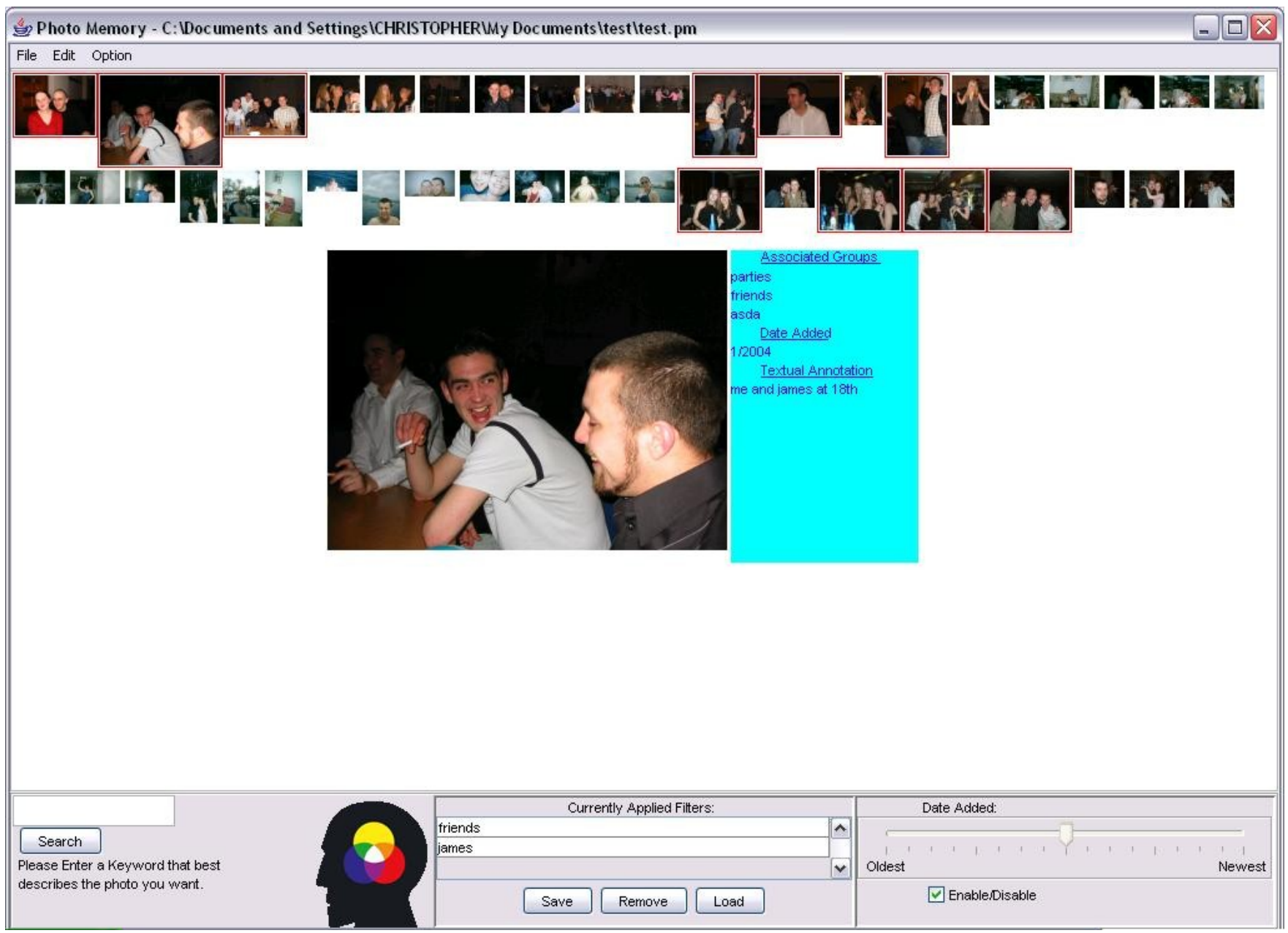

Figure 4: PhotoMemory User Interface, showing the Growing Paradigm and Feedback mechanism

\subsection{Filtering Options}

In section 6.1 we mentioned that in PhotoMemory many different types of recollection can be used to find images [design principle 1]. By providing a multi-dimensional form of interaction we can essentially help users utilize what they can remember to recover from other memory lapses. To make this possible a range of filter types can be applied to the collection to grow a particular set of images. These correspond to the contextual fragments identified by Elsweiler and others [2005] and are described below:

Visual Filtering: to exploit human visual perception mechanisms and strong recognition of visual stimuli users can skim and select photographs they deem appropriate. Photographs can also be grouped by filtering visually; combining annotation and retrieval phases. 
Semantic Filtering by free-text: recollected keywords can be used to filter photographs, although this relies on accurate recollection of the annotation process. Free-text filters match against both the names of groups that photographs are in and annotated descriptions.

Semantic Filtering by groups: by right-clicking on any thumbnail an option can be selected to filter the collection by groups associated with that image. The user is presented with a menu of checkboxes for each group and when a group is selected all of the images within that group are deemed appropriate.

Temporal Filtering via date line: a scrollbar is available that relates to a time-line. When the scrollbar is activated, images within a time frame close to that selected are deemed appropriate. This incorporates temporal recollections.

Spatial Filtering by screen location: to support accurate spatial recollection i.e. recollections of where images are located in relation to the screen or to other images, the PhotoMemory interface is designed so this information stays consistent throughout the search process.

Smart Filtering: Filters are applied and combined iteratively. Combinations can be saved and reapplied at later points in time. Saved filter combinations can be applied / removed in the same manner as the core filter types.

The smart filtering features also provide support for action-slips in PIM. When filters are applied a label representing that filter is added to the smart filtering component of the GUI [middle of the bottom panel in Figure 4]. These labels act as digital footprints representing the user's thoughts and actions. If, for instance, the user was to lose track of their thoughts while searching, they could examine the filters that they applied, establish why the display looks as it does and perhaps remember what they were trying to achieve by applying those filters [design principle 3]. 


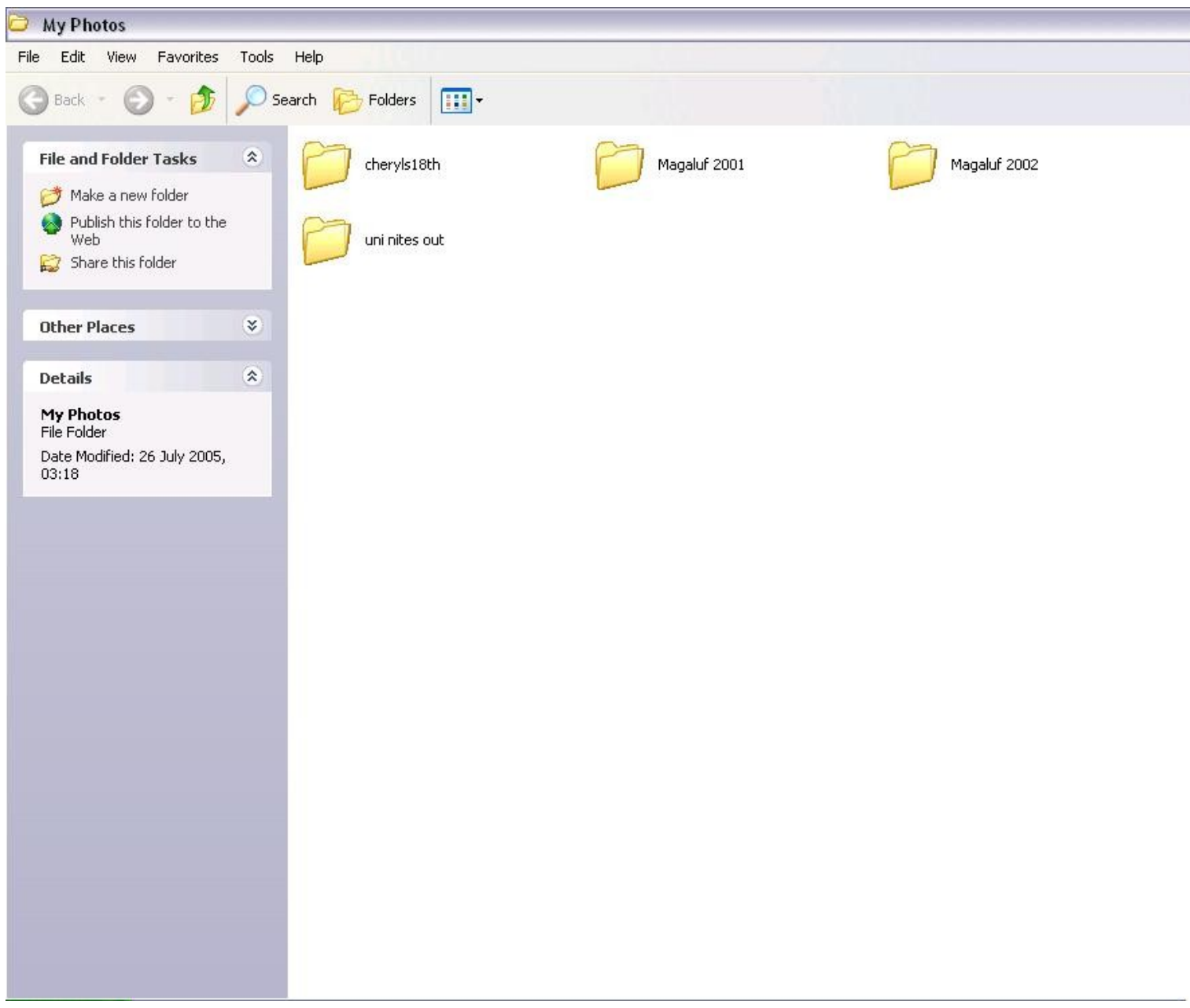

Figure 5: Standard hierarchical system (the folder structure in which participants organised their photographs 


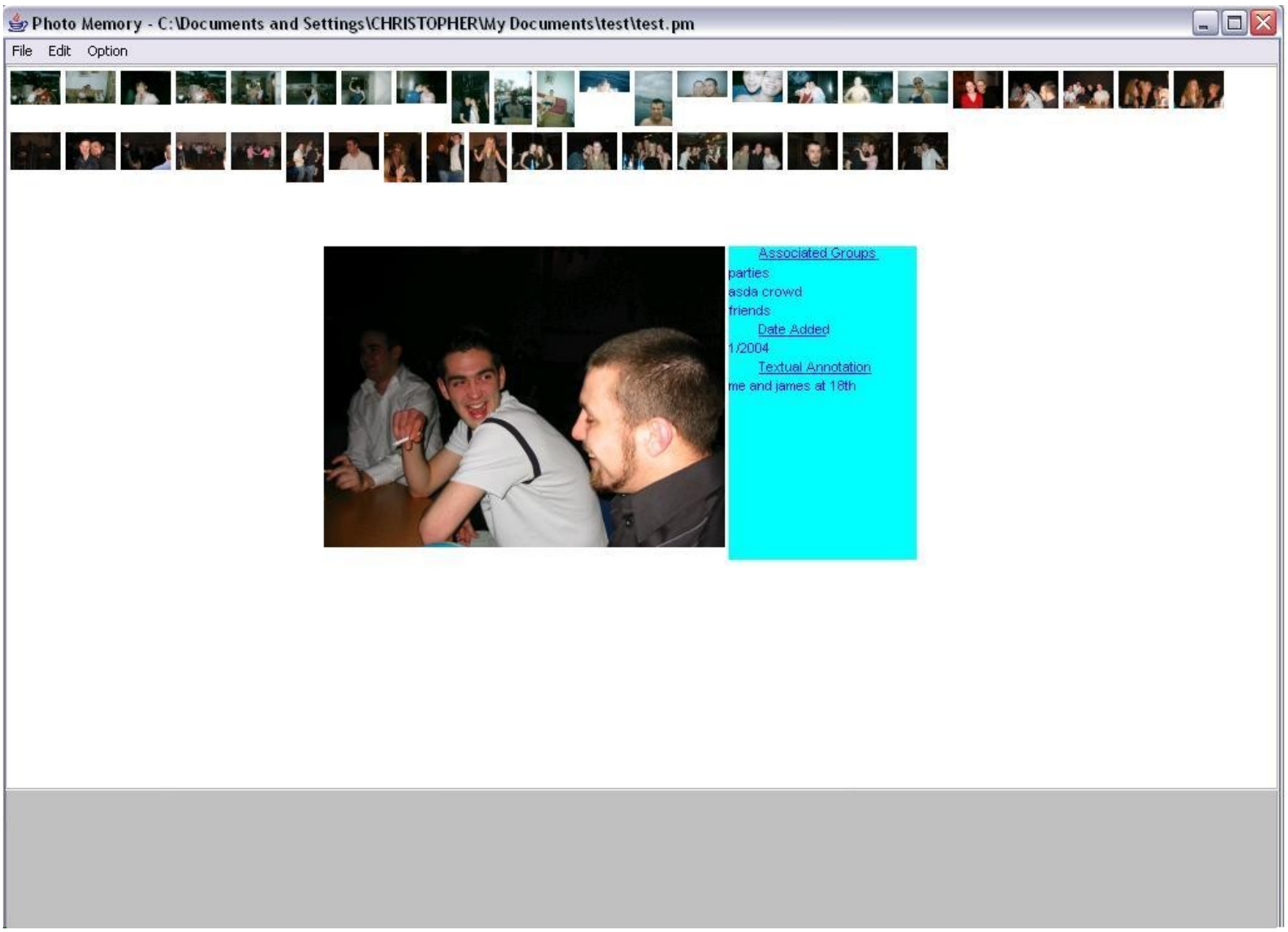

Figure 6: The restricted PhotoMemory interface with filtering facilities disabled

The PhotoMemory interface, therefore, has features to combat each of the lapse categories uncovered in the study of everyday memory problems. The interface is designed to facilitate re-finding personal photographs, which represent and remind the user of associated personal experiences that may have forgotten (retrospective lapses). Recovering the photograph from within the user's personal store can also be viewed as recovering from a retrospective lapse. The growing paradigm has a reminding function; reducing the chance that photographs will be forgotten about. Photographs may also act as reminders of future tasks (prospective lapses). Finally, the smart filtering facilities record the actions of the user in the form of applied filters - the display may provide the opportunity for the user to regain his / her train of thought if they are distracted from the search task. The interface also adheres to the five design principles outlined in section 5 . 


\section{Evaluating the Effectiveness of Multi-Dimensional Interaction}

To establish the usefulness of the memory supporting features, we tested the PhotoMemory interface (Figure 4) against a standard hierarchical system (the folder structure in which participants organised their photographs - Figure 5), as well as a restricted version of our software that used the same interface, but had the filtering features disabled (Figure 6). When using the restricted PhotoMemory interface the only means of searching was by scanning thumbnails and using the magnifying feedback feature. This system was included to determine if any benefits experienced when using PhotoMemory were simply a result of visual browsing.

The remainder of this paper describes a pilot study, which examined participants performing a series of realistic search tasks on the three systems described above. The aim was to extract indications about the usefulness of the features implementing the design principles from the memory study and attain design suggestions to improve our interface in future applications. The following sections describe our methodology and discuss some of the findings, relating their consequences to future work. In section 7.4 we evaluate the interfaces as search interfaces. The aim is to test the effectiveness of PhotoMemory as a search tool, using a more ubiquitous interface (folders) as a benchmark. In sections 7.5 and 7.6 we analyse PhotoMemory from point of view of the aims of interface - to support memory and facilitate its use in the search process.

\subsection{Participants}

Our evaluation involved 6 participants ( 1 female) who responded to an email advertisement within the Department of Computer and Information Sciences. The participants were all undergraduate computer science students with varying levels of expertise in photograph management. 3 of the participants described themselves as having good experience of the area, regularly taking and adding photographs and browsing their collections. 2 participants described themselves as having less experience of the technologies available to manage their collections, but still frequently add and browse. The last participant described their collection as being fairly static, but they tended to occasionally browse the collection.

Each participant provided a personal set of digital pictures, organised in a fashion that they determined themselves. This organisation represented System 2. The collections ranged from 106

images to 306, the average size was 207 pictures. The images were mainly organised hierarchically by events or time periods. Semantic information was rarely used to organise images, however, some folder names referred to image contents e.g. "Magaluf 2003". In the main, filenames were auto-generated by 
the camera or camera-phone with which they were taken, although some images had been renamed with meaningful, descriptive identifiers.

\subsection{Methods}

After a short demonstration, participants were given approximately 3 weeks to familiarise themselves with the two new systems, while creating and annotating their test collections. Participants were surveyed before the experiment to establish user characteristics and experience with photo management, during the experiment - pre-task, to gauge their recollection of photographs meeting task requirements and post-task, to determine their feelings towards each task and system. Finally, an exit questionnaire was issued to determine subject preferences across the three systems. While performing tasks users were encouraged to "think aloud". Asking the participants to verbalise their thoughts in this way provided the experimenters with an insight into what recollections were being used and why.

\subsection{Tasks}

When a user re-accesses photographs from their personal stores searches are generally of three main types [Rodden 1999]. Searching for

- Photographs from a particular event in the collection

- An individual photograph from the collection.

- Photographs that spanned across different events.

Our experimental tasks were created within these categories, tailored to suit each individual subject's collection. For example, type 1 tasks included find images from: "Leanne's $18^{\text {th }}$ Birthday party" and "one particular baseball game". Task 2 examples included "Find the image of you and Ross outside a church" and "Find the image of a Ferrari". Type 3 examples included "Find images from birthday parties", "images with friends" etc. Each subject performed 2 tasks of each type and the tasks and systems were rotated to minimise learning effects.

What we were doing by issuing these tasks was making participants aware of things they could not precisely remember i.e. a location or route to access a particular photograph or which photographs within their collections had specific attributes. Put another way, we were creating memory lapses for the participants to solve. This allowed us to evaluate how well our interface performed compared to existing systems and determine the usefulness of our implementation of the findings from the first study. 


\subsection{Performance of Systems}

The time taken to perform each task, the number of images retrieved to complete the task and how the retrieved image set matched original recollections (Scale 1-5) were recorded and used to objectively and subjectively compare the performance of the systems. These data are summarized in Table 3: Objective data recorded during the study (best value in bold).

Table 3: Objective data recorded during the study (best value in bold)

\begin{tabular}{|c|c|c|c|}
\hline Task & PhotoMemory & Folders & $\begin{array}{c}\text { Restricted } \\
\text { PhotoMemory }\end{array}$ \\
\hline Task 1 & & & \\
\hline Time to complete task (secs) & 116.50 & $\mathbf{1 0 0 . 0 0}$ & 154.25 \\
\hline No. images retrieved & 12.00 & 12.00 & $\mathbf{1 2 . 7 5}$ \\
\hline Selected group accurately reflected recollections & $\mathbf{1 . 7 5}$ & 3.25 & 3.25 \\
\hline Task 2 & & & 110.25 \\
\hline Time to complete task (secs) & $\mathbf{3 5 . 2 5}$ & 47.00 & 1.00 \\
\hline No. images retrieved & 1.00 & 1.00 & $\mathbf{1 . 0 0}$ \\
\hline Selected group accurately reflected recollections & $\mathbf{1 . 0 0}$ & 2.50 & 215.00 \\
\hline Task 3 & & & 16.25 \\
\hline Time to complete task (secs) & $\mathbf{1 4 5 . 2 5}$ & 146.00 & 4.25 \\
\hline No. images retrieved & $\mathbf{2 4 . 2 5}$ & 20.75 & \\
\hline Selected group accurately reflected recollections & $\mathbf{2 . 7 5}$ & 3.00 & 159.83 \\
\hline Average for All Tasks & & & 10.00 \\
\hline Time to complete task (secs) & 99.00 & $\mathbf{9 7 . 6 7}$ & 2.83 \\
\hline No. images retrieved & $\mathbf{1 2 . 4 2}$ & 11.25 & 2.92 \\
\hline Selected group accurately reflected recollections & $\mathbf{1 . 8 3}$ & & \\
\hline
\end{tabular}

Table 4: Subjective Preferences from Exit Questionnaire (best value in bold)

\begin{tabular}{|lccc|}
\hline & PhotoMemory & Folders & $\begin{array}{c}\text { Restricted } \\
\text { PhotoMemory }\end{array}$ \\
\hline Preferred System & $\mathbf{6}$ & 0 & 0 \\
Easiest to system to use & $\mathbf{3}$ & 2 & 1 \\
Fastest System & $\mathbf{6}$ & 0 & 0 \\
Most effective when searching for one photograph & $\mathbf{6}$ & 0 & 0 \\
Most effective when searching for multiple photographs & $\mathbf{6}$ & 0 & 0 \\
\hline
\end{tabular}


The mean search completion times for the three systems were 99s for PhotoMemory, 97.7s for the restricted version of PhotoMemory, and 159s for the hierarchical system. On average the restricted version of PhotoMemory was fastest. However, the unrestricted PhotoMemory was on average faster for tasks 2 and 3. It makes sense that our interfaces were faster for task type 3, which involved searching for images spanning different events, as it cuts across the temporal boundaries defined within many of the hierarchies.

When examining the subjective ratings of how recovered image sets match pre-task recollections (scale 1-5), we found that image sets returned when using PhotoMemory (mean=1.83 stdev=0.99) tended to match memories less than those in the restricted version (mean=2.92 stdev=1.38) and hierarchical folders $($ mean=2.83 stdev=1.57). From observing and interviewing participants we discovered that this was usually a positive outcome and meant larger result sets were obtained than those anticipated from recollections alone. One explanation for this could be that using the multi-dimensional interface facilitated the acquisition of additional cues that allowed images to be found that were not cued by the task.

From the exit survey data [Table 4], we can clearly see that the preferred system was PhotoMemory. All of the participants deemed PhotoMemory to be their favourite system. Further, contradicting the timed data; participants rated PhotoMemory as the fastest system. It was also judged to be the most effective when searching for both single and multiple images. The only category that the multidimensional interface was not deemed completely superior was in terms of ease of use. This is perhaps related to the fact that the method of interaction is new and unfamiliar to users and using the system requires the user to make more decisions during retrieval.

\subsection{Observed Behaviour}

The following sections describe the recollections participants offered in the pre-task surveys, observed participant search behaviour and attempts to rationalise the reasons behind this behaviour. We identify which features of photographs were remembered and examine if these influenced the users' search strategies.

\subsubsection{Recollected Features}

The features of photographs that the participants remembered largely mapped to the contextual fragments as described in Elsweiler and others [2005]. Consequently, in this section we only restate the main points and emphasize any supplementary findings. 
What participants tended to remember about their images were "fragments of context". These corresponded to either 1) the visual context: descriptions of what was visually present in the photograph e.g. "four of my friends by a house". 2) Surrounding context: descriptions of the reasons why the photograph was taken or the details about the surrounding event. 3) External context: descriptions of manipulations or features of the photograph itself rather than the content e.g. annotations applied to the photographs, the fact that the photograph was of poor quality or the type of camera used etc.

The descriptions of memories were often highly visual. For example, "rustic pink coloured railings with snow lining the top". Environmental details were frequently mentioned, including weather and location information. Personal experiences tended to be accentuated when describing recollected images, possibly indicating that personal connections to images strengthen memories and allow descriptions to be given in extra detail. Further, personal feelings and emotions repeatedly formed part of recalled descriptions e.g. "We were so cold and tired". We feel that these personal aspects should be utilised in some way in order to improve re-access. Additionally, images were often referred to in terms of their relationship to each other e.g. "one was earlier - it was warmer and sunnier, while another was later - you can see that we were getting cold". One participant had expert recollection of the technical features of photos, including their size, resolution and the camera with which they were taken. This information directly affected the way in which he searched. For example, when using the folder system (system 2), he often sorted files by their file size or file extension, based on the recollection of the camera used.

Overall, the features recollected appeared to influence the participants' search strategies. Nevertheless, not every aspect of the recollected data was used during searches. The following section details the way participants searched and the features of PhotoMemory that were used, relating them to the contextual information supplied before the search task commenced.

\subsubsection{Features of PhotoMemory that were used}

From the same starting point i.e. the same recollected features, participants' search behaviour changed with different systems. They used different types of recollected fragment based on the system in use. Across all systems, however, it was observed that completing individual tasks involved several bursts of searching. During these bursts, participants tended to focus on a single fragment associated with an image(s) that they had remembered and this determined their search strategy. Only when a search burst failed did they decide to use other recollected features. It was extremely rare for users to 
utilise multiple aspects of context within a single burst of searching. Only when using PhotoMemory was this evident and even then only when another image triggered improved recollection.

When using PhotoMemory the common practise was to start searches with keyword filters, utilising semantic recollections. This differed from the hierarchical and restricted systems as they provide few features to exploit semantic contextual information. Group filtering was also used, although, not as frequently as keyword searches. One explanation for this could be that the feature required the user to right click on an image before filtering; hiding the feature from the user. PhotoMemory's date filter feature was used very sparingly. Nevertheless, temporal context was used in different ways. For instance, because PhotoMemory orders images temporally, participants identified key images for a recollected time period and browsed around them, effectively filtering by date themselves.

When searching with PhotoMemory there was evidence of increasing recollection of desired photographs as the search continued and the extra information altering search behaviour i.e. users made use of the retrieval journey concepts discussed in [Section 6.1]. Users started searches using small pieces of information about the photographs they wish to find; these recollections orientated them along their journey. Through interaction with the system, additional information was acquired or recollected, resulting in more detailed search aims and improved awareness of the information space with relation to these aims. For example, if a user was asked to find a photo of a particular friend (John). He may start searching using "John" as a keyword. When this process fails to return an appropriate image (poor annotation), the user may recall a particular experience he shared with John and browse the collection looking for images of that experience. While browsing the user may find images of a football game, which trigger a memory of another time when he and John watched football together shortly after the user had bought a new camera phone. He may remember that all of his camera phone images had been semantically grouped and apply a filter based on this. As he knew images would be early (he had just bought the phone) the user would find an image of John when they watched the football match. There was no evidence of such progression of knowledge and developing strategies in either the restricted version of PhotoMemory or the folders.

It also appeared that, when using PhotoMemory, filtering interaction was used to create dynamic groupings of images. Participants referred to the sets as having shared properties and in this way they were treated in similar means to photographs within a hierarchical folder. For example, "all of those images: are of Colin.... contain scenery... are from nights out" etc.. The difference between this dynamic 
grouping behaviour and using standard folders is there is no dependency on the precise location within a hierarchy to retrieve particular image(s) from the groups.

When using the restricted version of PhotoMemory (system 3), users were limited to exploiting visual features alone. This essentially meant mousing over each image until a match was found. This style of interaction tended to frustrate users, with 4 out of 6 subjects remarking that they felt uncomfortable performing searches in this way. One participant stated: "I know that it was when I was in the States, but that doesn't help here".

Subjects' behaviour and performance when using their hierarchical folder system was influenced not only by recollection of the photograph(s) they wish to find, but by their knowledge of their hierarchical structure. Subjects who understood the spatial organisation tended to find images quickly, while others found it difficult to narrow search domain at all. Further, the experts could utilise varying image properties to find images such as sorting images by date or size, while those with little understanding of the information space or file systems relied on folder and file names that were not always present or meaningful.

In the main, we found that the use of recalled context within searches was limited to a small sub-set of that actually remembered and very rarely were multiple aspects used in one search. Users like the idea of exploiting the additional information to their advantage and show signs of practising this when the facilities are made available. Further, search performance when using PhotoMemory did not rely as heavily on expert knowledge of the information space - as was the case for the hierarchical systems. Despite these benefits, work must be done to assist this process by allowing users to interact with the system in a natural fashion while using multiple aspects of context.

\subsection{The Findings in Relation to Lapses in Memory}

In this section we relate the findings of the pilot to the three primary lapse types discussed in part 1 of this article.

Shorter search times, yielding larger result sets and strong user preference for PhotoMemory over traditional folders suggests that PhotoMemory provides assistance in overcoming retrospective information lapses associated with PIM. As the core aim of PIM is to facilitate re-access to information and promote information re-use, these findings are of greatest importance to our work. Further the frequency with which retrospective, information-based lapses were recorded in our general study of memory failure indicates a need for tools such as PhotoMemory for different kinds of information objects. 
The discovery that PhotoMemory tended to yield larger result sets also has implications for solving prospective memory lapses. Interviews with participants confirmed that large result sets were a positive outcome and that using PhotoMemory allowed images to be retrieved that participants may not have otherwise remembered. It is, however, unclear if any single feature of PhotoMemory was responsible for this effect or whether it was a combined effect in the interface as a whole.

Our method of evaluation did not allow successful recovery from action slips to be detected. Certainly, in our tests, participants made no use of the smart filtering facilities in the trials and did not refer to using the display of previously applied filters as a memory jogger. However, as participants were focused on one task at a time in an environment without interruptions, we feel this was an expected outcome. Evaluations, such as in [Dumais et al. 2003], where participants used the tool in "the wild" would be a better means to evaluate PhotoMemory's potential for assisting with action slips.

\section{Conclusions and Future Work}

In part 1 of this article we described a three-component study performed to discover the variety and frequencies of memory problems experienced in a week by a wide range of individuals. The motivation for this work was the observation by the authors and other researchers that memory lapses hinder PIM and that similarities exist between PIM lapses and other everyday memory lapses. We performed the study to gain additional information to assist the design of supportive user interface tools for managing personal information. The results were presented and an analysis provided of the challenges that users

face when recovering from such lapses. Our result set illustrates various causal situations for memory lapses and provides an insight into the strategies we use to prevent and recover from lapses. From our results we presented a series of design principles that we hypothesized would improve the design of digital PIM tool interfaces.

Building on this work, in part 2 of the article, we evaluated a tool designed to illustrate our principles. The tool abandoned design metaphors commonly associated with photograph management software in favour of an interface which supported the three types of memory lapse uncovered in the first study. The interface allowed multi-modal access to photographs and attempted to re-create the contexts in which photos were previously viewed and annotated. We also looked at how contextual fragments of recollection are used in real search situations. The results show that although users are normally able to remember several characteristics of photographs they wish to find, in practise they use very few of these during a typical search process for personal photographs. Nevertheless, we found that users like the idea 
of using multiple fragments of recollection in an iterative manner, and we observed greater use of multiple aspects of context when they used PhotoMemory - an interface specifically designed to encourage this.

The work presented in this article suggests that the type of memory-oriented interaction promoted by PhotoMemory is successful for photographs: users liked the interface and showed improved ability to refind personal images when compared to the other interfaces tested. However, photographs are highly visual and are associated with a wide range of memory types; they have clear links to episodes, experiences, people, places etc. This may make annotated photographs particularly suited to our browsing-searching hybrid. We are currently building on our findings by exploring similar interfaces for other types of information object that are less visual, such as email messages and objects that do not have a recognised store, such as web pages.

\section{References}

Adar, E.; D.Kargar \& Stein, L.A. (1999),Haystack: per-user information environments, in 'CIKM '99: Proceedings of the eighth international conference on Information and knowledge management', ACM Press, New York, NY, USA, pp. 413--422.

Bederson, B.B. (2001),'PhotoMesa: a zoomable image browser using quantum treemaps and bubblemaps"UIST '01: Proceedings of the 14th annual ACM symposium on User interface software and technology', ACM Press, New York, NY, USA, 71--80.

Bower, G.; Schill, S.T. \& Tulving, E. (1994), 'Reducing retroactive interference: An interference analysis', Journal of Experimental Psychology: Learning, Memory \&amp; Cognition 20, 51-66.

Brown, R. \& McNeil, D. (1966), ' The "tip of the tongue" phenomenon', The "tip of the tongue" phenomenon. Journal of Verbal Learning and Verbal BehaviorJournal of Verbal Learning and Verbal Behavior 5, 325-337..

Bruce, H. (2005), 'Personal anticipated information need', Information Research 10(3), 232.

Bruce, H.; Jones, W. \& Dumais, S. (2004), 'Information behaviour that keeps found things found', Information Research $\mathbf{1 0 .}$

Byström, K. \& Järvelin, K. (1995), 'Task complexity affects information seeking and use', Information Processing and Management 31(2), 191-213.. 
Capra, R.G. \& Quiñones, M.A.P. (2005), 'Using Web Search Engines to Find and Refind Information', Computer 38(10), 36--42.

Capra, R.G. \& Quiñones, M.A.P. (2003), 'Re-Finding Found Things: An Exploratory Study of How Users Re-Find Information', .

Carroll, J. (1982), 'Creative names for personal files in an interactive computing environment', International Journal of Man-Machine Studies 16, 405-438..

Case, D. (1991), 'Conceptual organization and retrieval of text by historians: The role of memory and metaphor.', JASIST 42(9), 657-668.

Clark, H.H. \& Clark, E.\&amp; Jovanovich., H.B., ed. (1977), Psychology and language, New York.

Cohen, G. (2004), Memory in the Real World, Psychology Press.

Crovitz, H.F. \& Daniel, W. (1984), 'Measurement of everyday memory: toward the prevention of forgetting', Bulletin of Psychonomic Society 22, ..

Czerwinski, M.; Chrisman, S. \& Schumacher, C. (1991), 'the effects of warnings and display similarities on interruptions in multi-tasking environments', SIGCHI Bulletin 23, 38-39.

Czerwinski, M. \& Horvitz, E. (2002),An Investigation of Memory for Daily Computing Events, in 'Proceedings of HCI 2002', pp. 230-245.

Dey, A. \& Abowd, G. (2000), 'CybreMinder: A Context-Aware System for Supporting Reminders', Proceedings of the 2nd international symposium on Handheld and Ubiquitous Computing, 172-186.

Dourish, P.; Edwards, W.K.; LaMarca, A.; Lamping, J.; Petersen, K.; Salisbury, M.; Terry, D.B. \& Thornton, J. (2000), 'Extending document management systems with user-specific active properties', ACM Trans. Inf. Syst. 18(2), 140--170.

Ducheneaut, N. \& Bellotti, V. (2001), 'E-mail as habitat: an exploration of embedded personal information management', interactions 8(5), 30--38.

Dumais, S.; Cutrell, E.; Cadiz, J.; Jancke, G.; Sarin, R. \& Robbins, D. (2003),Stuff I've seen: a system for personal information retrieval and re-use, in 'SIGIR '03: Proc. 26th annual international ACM SIGIR conference on Research and development in informaion retrieval', ACM Press, New York, NY, USA, pp. 72--79. 
Ebbinghaus, H. (1885), Über das Gedchtnis. Untersuchungen zur experimentellen Psychologie, Leipzig: Duncker \&amp; Humblot.

Eldridge, M.; Sellen, A. \&amp; Bekerian, D. (1992),'The range, frequency, and severity of memory problems at work'(EPC-92-129, ), Technical report, EuroPARC.

Elsweiler, D.; Ruthven, I. \&amp; Jones, C. (2005),'Dealing with fragmented recollection of context in information management"Context-Based Information Retrieval (CIR-05) Workshop in Fifth International and Interdisciplinary Conference on Modeling and Using Context (CONTEXT-05)'.

Eysenck, M.W. (2001), Principles of Cognitive Psychology, Psychology Press.

Freeman, E. \& Gelernter, D. (1996), 'Lifestreams: a storage model for personal data', SIGMOD Rec. 25(1), 80--86.

Gifford, D.; Jouvelot, P.; Sheldon, M.A. \& Jr., J.W.O. (1991),Semantic file systems, in 'SOSP '91: Proceedings of the thirteenth ACM symposium on Operating systems principles', ACM Press, New York, NY, USA, pp. 16--25.

Gwizdka, J. (2000),'Timely Reminders: A Case Study of Temporal Guidance in PIM and Email Tools Usage"CHI'2000'.

Hayes, P.; Andersen, P.; Nirenburg, I. \& Schmandt, L. (1990), 'TCS: a shell for content-based text categorization', Artificial Intelligence Applications, 1990., Sixth Conference on, 320-326.

Heesch, D. \& Rüger, S. (2004),NNk networks for content-based image retrieval, in 'ECIR', LNCSSpringer, , pp. $253-266$.

Herrmann, D.J. (1982), 'Know thy memory: The use of questionnaires to assess and study memory', Psychological Bulletin 92(2), 434-452.

Herrmann, D.J. \& Neisser, U. (1978), 'An inventory of everyday memory experiences', $M M$ Gruneberg, PE Morris, \&amp; RN SykesEds, 35--51.

Hertzum, M. \& Pejtersen, A. (2000), 'The information-seeking practices of engineers: searching for documents as well as for people', Information Processing and Management 36(5), 761-778.

Hightower, R.; Ring, L.; Helfman, J.; Bederson, B. \& Hollan, J. (1998), Graphical multiscale web histories: A study of padprints, in 'Hypertext'98', ACM Press, , pp. 58-65. 
Jones, S.R. \& Thomas, P. (1997), 'Empirical assessment of individuals' 'personal information management systems", Behaviour and Information Technology 16, 158-160..

Jones, W.; Bruce, H. \& Dumais, S. (2003), 'How do people get back to information on the web? How can they do it better', 9th IFIP TC13 International Conference on Human-Computer Interaction (INTERACT 2003), Zurich, Switzerland.

Jones, W.; Bruce, H.; Foxley, A. \&amp; Munat, C. (2005),'The Universal Labeler: Plan the Project and Let Your Information Follow"ASIST '05'.

Jones, W.; Dumais, S. \& Bruce, H. (2002),Once found, what then? A study of ?keeping? behaviors in the personal use of Web information, in 'Proceedings of the American Society for Information Science and Technology', pp. 391--402.

Karger, D.R.; Katz, B.; Lin, J. \& Quan, D. (2003),Sticky notes for the semantic web, in 'IUI '03: Proceedings of the 8th international conference on Intelligent user interfaces', ACM Press, New York, NY, USA, pp. 254--256.

Krishnan, A. \& Jones, S. (2005), 'TimeSpace: activity-based temporal visualisation of personal information spaces', Personal and Ubiquitous Computing 9(1), 46--65.

Kwasnik, B. (1989),'The influence of context on classificatory behavior', $\mathrm{PhD}$ thesis, Rutgers, the State University of New Jersey.

Kwasnik, B. (1989), 'How a personal document's intended use or purpose affects its classification in an office', SIGIR Forum 23(SI), 207--210.

Lamming, M.; Brown, P.; Carter, K.; Eldridge, M.; Flynn, M.; Louie, G.; Robinson, P. \& Sellen, A. (1994), 'The Design of a Human Memory Prosthesis', The Computer Journal 37(3), 153-163.

Lamming, M. \& Flynn, M. (1994),Forget-me-not: Intimate computing in support of human memory, in 'Proceedings of FRIEND21,'.

Lansdale, M. (1988), 'The psychology of personal information management.', Appl Ergon 19(1), 5566.

Loftus, E. (1979), Eyewitness Testimony, Cambridge, MA: Harvard University Press..

Loftus, E.; Smith, K.; Johnson, D. \& Fiedler, J. (1988), Practical aspects of memory: Current research and issues, Wiley, chapter Remembering when: Errors in the dating of autobiographical memories. 
Malone, T. (1983), 'How do people organize their desks?: Implications for the design of office information systems', ACM Trans. Inf. Syst. 1(1), 99--112.

Marshall, C. (1998),Toward an ecology of hypertext annotation, in 'HYPERTEXT '98: Proceedings of the ninth ACM conference on Hypertext and hypermedia : links, objects, time and space---structure in hypermedia systems', ACM Press, New York, NY, USA, pp. 40--49.

Mills, T.; Pye, D.; Sinclair, D. \&amp; Wood, K. (2000),'Shoebox: A digital photo management system', Technical report, AT\&T Laboratories, Cambridge.

Neisser, U. (1982), Memory Observed: Remembering in Natural Contexts, Freedman \&amp; Co. 1982.

Palen, L. \& Salzman, M. (2002),Voice-mail diary studies for naturalistic data capture under mobile conditions, in ' $\mathrm{CSCW}$ '02: Proceedings of the 2002 ACM conference on Computer supported cooperative work', ACM Press, New York, NY, USA, pp. 87--95.

Platt, J.; Czerwinski, M. \& Field, B. (2003), 'PhotoTOC: automatic clustering for browsing personal photographs', Information, Communications and Signal Processing, 2003 and the Fourth Pacific Rim Conference on Multimedia. Proceedings of the 2003 Joint Conference of the Fourth International Conference on 1, 6-10.

R.Boardman \& A.Sasse, M. (2004),"Stuff goes into the computer and doesn't come out": a cross-tool study of personal information management, in 'CHI '04: Proceedings of the SIGCHI conference on Human factors in computing systems', ACM Press, New York, NY, USA, pp. 583--590.

Reason, J. (1990), Human Error, Cambridge University Press.

Rekimoto, J. (1999),Time-machine computing: a time-centric approach for the information environment, in 'UIST '99: Proceedings of the 12th annual ACM symposium on User interface software and technology', ACM Press, New York, NY, USA, pp. 45--54.

Renaud, K. (2000),'Expediting Rapid Recovery from Interruptions by Providing a Visualisation of Application Activity', in C Paris; N Ozkan \&amp; S Howard ans S Lu, ed.,'OZCHI 2000. Interfacing Reality in the New Millennium', Univ. of Technology Sydney, Sydney Australia, 348-355.

Rieman, J. (1993), The Diary Study: A Workplace-Oriented Research Tool to Guide Laboratory Efforts Collecting User-Information for System Design., in 'INTERCHI'93', pp. 321-326. 
Rodden, K. (1999),How do people organise their photographs, in 'BCS IRSG 21st Annual Colloquium on Information Retrieval Research,Glasgow, Scotland'.

Rodden, K.; Basalaj, W.; D.Sinclair \& Wood, K. (2001),Does organisation by similarity assist image browsing?, in 'CHI '01: Proceedings of the SIGCHI conference on Human factors in computing systems', ACM Press, New York, NY, USA, pp. 190--197.

Rubin, D. (1977), 'Very long-memory for prose and verse', Journal of Verbal Learning and Verbal Behavior 16, 611-621.

Rubin, D. \& Wenzel, A. (1996), 'One Hundred Years Of Forgetting: A quantitative description of retention', Psychological Bulletin 103, 734-760.

Rubinstein, J.; Meyer, D. \& Evans, J. (2001), 'Executive Control of Cognitive Processes in Task Switching', Journal of Experimental Psychology: Human Perception and Performance 27(4), 763-797.

Sachs, J.S. (1967), 'Recognition memory for syntactic and semantic aspects of connected discourse', Percept. Psychophys 2, 437-42.

Spink, A. \& Park, M. (2004), 'Information Retrieval as Multitasking: An Exploratory Framework', ACM SIGIR 2004 Workshop on 'Information Retrieval in Context, 16--19.

Sunderland, A.; Harris, J. \& Baddeley, A. (1983), 'Do laboratory tests predict everyday memory?', Journal of Verbal Learning and Verbal Behavior 22, 341-357.

Teevan, J.; Alvarado, C.; Ackerman, M.S. \& Karger, D.R. (2004), The perfect search engine is not enough: a study of orienteering behavior in directed search, in 'CHI '04: Proceedings of the SIGCHI conference on Human factors in computing systems', ACM Press, New York, NY, USA, pp. 415--422.

Terry, W. (1988), 'Everyday forgetting: Data from a diary study', Psychological Reports 62, 299-303.

Whittaker, S. \& Sidner, C. (1996),Email overload: exploring personal information management of email, in 'CHI '96: Proceedings of the SIGCHI conference on Human factors in computing systems', ACM Press, New York, NY, USA, pp. 276--283.

Yang, Y. (1994),Expert network: effective and efficient learning from human decisions in text categorization and retrieval, in 'SIGIR '94: Proceedings of the 17th annual international ACM SIGIR conference on Research and development in information retrieval', Springer-Verlag New York, Inc., New York, NY, USA, pp. 13--22. 
Yee, K.; Swearingen, K.; Li, K. \& Hearst, M. (2003),Faceted metadata for image search and browsing, in 'CHI '03: Proceedings of the SIGCHI conference on Human factors in computing systems', ACM Press, New York, NY, USA, pp. 401--408. 


\section{Appendix A - Memory Questionnaire}

\section{Personal Information}

\begin{tabular}{|l|l|}
\hline Name: & \\
\hline Age: & \\
\hline Sex: & Male / Female \\
\hline Education Level: & School (Standard / O Grade) \\
& School (Higher / A Grade) \\
& College (HNC) \\
& College (HND) \\
& University (Undergraduate Degree) \\
& University (Post-Graduate Degree) \\
Other \\
\hline Job Title & \\
\hline $\begin{array}{l}\text { Would you describe yourself as an information } \\
\text { worker? }\end{array}$ & Yes / No \\
\hline Do you use a computer? & Yes / No \\
\hline
\end{tabular}

\section{Reading Habits}

\begin{tabular}{|l|l|}
\hline How often do you read generally? & Never $\square$ Rarely $\square$ Monthly $\square$ Weekly $\square$ Daily $\square$ \\
\hline How often do you read books? & Never $\square$ Rarely $\square$ Monthly $\square$ Weekly $\square$ Daily $\square$ \\
\hline How often do you read newspapers? & Never $\square$ Rarely $\square$ Monthly $\square$ Weekly $\square$ Daily $\square$ \\
\hline How often do you read magazines? & Never $\square$ Rarely $\square$ Monthly $\square$ Weekly $\square$ Daily $\square$ \\
\hline How often do you read journals? & Never $\square$ Rarely $\square$ Monthly $\square$ Weekly $\square$ Daily $\square$ \\
\hline
\end{tabular}

\section{Communication Habits}

On average how many of the following to you receive each day?

\begin{tabular}{|l|l|}
\hline Email Messages & None $\square$ 0-15 $\square$ 16-30 $\square$ 31-50 $\square 50+\square$ \\
\hline Telephone Calls & None $\square$ 0-5 $\square$ 5-10 $\square$ 10-15 $\square 15+\square$ \\
\hline Text Messages & None $\square$ 0-15 $\square$ 16-30 $\square$ 31-50 $\square 50+\square$ \\
\hline Instant messages (Msn, AOL, etc) & None $\square$ 0-15 $\square$ 16-30 $\square$ 31-50 $\square 50+\square$ \\
\hline Traditional Mail & None $\square$ 0-5 $\square$ 5-10 $\square$ 10-15 $\square 15+\square$ \\
\hline Faxes & None $\square$ 0-5 $\square$ 5-10 $\square$ 10-15 $\square 15+\square$ \\
\hline $\begin{array}{l}\text { How often do you meet people during the } \\
\text { day? }\end{array}$ & None $\square$ 0-15 $\square$ 16-30 $\square$ 31-50 $\square 50+\square$ \\
\hline
\end{tabular}

\section{Internet Browsing Habits}

\begin{tabular}{|l|l|l|}
\hline How often do you use the internet? & Never $\square$ Rarely $\square$ Weekly $\square$ Daily $\square$ Many times a day $\square$ \\
\hline What do you use the Internet for? & Work & $\cdot$ \\
& Research & $\cdot$ \\
& Shopping & $\cdot$ \\
& Comparing Prices & $\cdot$ \\
& Music & $\cdot$ \\
& Hobbies & $\cdot$ \\
& News & \\
\hline & Other & \\
\hline
\end{tabular}




\section{Organisation}

\begin{tabular}{|l|l}
\hline Would you describe yourself as an organised person? & Yes / No \\
\hline
\end{tabular}

\begin{tabular}{l|l|llllll} 
On a scale of 1-5 how organised are you? & (Not at all) & $\mathbf{1}$ & $\mathbf{2}$ & $\mathbf{3}$ & $\mathbf{4}$ & $\mathbf{5}$ & (Very)
\end{tabular}

\begin{tabular}{|l|l}
\hline Do you like established routines? & Yes / No
\end{tabular}

\begin{tabular}{|c|c|}
\hline \multirow{2}{*}{$\begin{array}{l}\text { How organised is your music collection? } \\
\text { Describe the method you use to organise your mu- } \\
\text { sic. }\end{array}$} & $\begin{array}{llllll}\text { (Not at all) } & \mathbf{1} & \mathbf{2} & \mathbf{3} & \mathbf{4} & \mathbf{5} \text { (Very) } \\
\end{array}$ \\
\hline & \\
\hline How organised is your photograph collection? & $\begin{array}{llllll}\text { (Not at all) } & \mathbf{1} & \mathbf{2} & \mathbf{3} & \mathbf{4} & \mathbf{5} \text { (Very) }\end{array}$ \\
\hline $\begin{array}{l}\text { Describe the method you use to organise your } \\
\text { photographs. }\end{array}$ & \\
\hline How organised are the files on your PC? & $\begin{array}{llllll}\text { (Not at all) } & \mathbf{1} & \mathbf{2} & \mathbf{3} & \mathbf{4} & \mathbf{5} \text { (Very) }\end{array}$ \\
\hline $\begin{array}{l}\text { Describe the method you use to organise your } \\
\text { files. }\end{array}$ & \\
\hline How organised are your web bookmarks? & $\begin{array}{llllll}\text { (Not at all) } & \mathbf{1} & \mathbf{2} & \mathbf{3} & \mathbf{4} & \mathbf{5} \text { (Very) } \\
\end{array}$ \\
\hline $\begin{array}{l}\text { Describe the method you use to organise your } \\
\text { bookmarks. }\end{array}$ & \\
\hline
\end{tabular}




\section{Memory Performance}

\begin{tabular}{|l|l|}
\hline $\begin{array}{l}\text { How would you rate your memory perform- } \\
\text { ance generally? }\end{array}$ & Very Poor $\square$ Poor $\square$ Average $\square$ Good $\square$ Exceptional $\square$ \\
\hline
\end{tabular}

On a scale of 1-5 how often do the following situations occur to you? $(1=$ never, $2=$ rarely, $3=$ monthly, $4=$ weekly, $5=$ daily)

Retrospective Memory lapses

\begin{tabular}{|l|l|}
\hline Forget a person's name & $1 \square 2 \square 3 \square 4 \square 5 \square$ \\
\hline Forget a once known fact & $1 \square 2 \square 3 \square 4 \square 5 \square$ \\
\hline Misplace an item such as keys or mobile phone & $1 \square 2 \square 3 \square 4 \square 5 \square$ \\
\hline Forget a word or spelling of a word & $1 \square 2 \square 3 \square 4 \square 5 \square$ \\
\hline Forget where you read something & $1 \square 2 \square 3 \square 4 \square 5 \square$ \\
\hline Forget items on a list & $1 \square 2 \square 3 \square 4 \square 5 \square$ \\
\hline Forget specific details of read material and have to look it up or re-read & $1 \square 2 \square 3 \square 4 \square 5 \square$ \\
\hline Have to spend time searching for a file or information stored on your PC & $1 \square 2 \square 3 \square 4 \square 5 \square$ \\
\hline Forget the address of a webpage & $1 \square 2 \square 3 \square 4 \square 5 \square$ \\
\hline $\begin{array}{l}\text { Confuse the content of websites e.g. think that you read something on a web- } \\
\text { site when you actually read it on another webpage with similar content }\end{array}$ & $1 \square 2 \square 3 \square 4 \square 5 \square$ \\
\hline Forget about a past experience & $1 \square 2 \square 3 \square 4 \square 5 \square$ \\
\hline Forget where you were when an event happened & $1 \square \square \square 3 \square 4 \square 5 \square$ \\
\hline
\end{tabular}

Prospective Memory Lapses

\begin{tabular}{|l|l|}
\hline Forget about an appointment & $1 \square 2 \square 3 \square 4 \square 5 \square$ \\
\hline Forget to pay a bill or similar task & $1 \square 2 \square 3 \square 4 \square 5 \square$ \\
\hline Forget to pass on a message to someone & $1 \square 2 \square 3 \square 4 \square 5 \square$ \\
\hline Forget to bring something with you & $1 \square 2 \square 3 \square 4 \square 5 \square$ \\
\hline Forget about a special date such as a birthday or anniversary & $1 \square 2 \square 3 \square 4 \square 5 \square$ \\
\hline
\end{tabular}

Action Slips

\begin{tabular}{|l|c|}
\hline Forget to complete a task after being interrupted & $1 \square 2 \square 3 \square 4 \square 5 \square$ \\
\hline $\begin{array}{l}\text { Miss out a step in a procedure e.g. sending an email without adding an attach- } \\
\text { ment or forgetting to put sugar in a cup of tea }\end{array}$ & $1 \square 2 \square 3 \square 4 \square 5 \square$ \\
\hline $\begin{array}{l}\text { Forget the intention of an action e.g. go upstairs to do something and when } \\
\text { you arrive forget what the task was }\end{array}$ & $1 \square 2 \square 3 \square 4 \square 5 \square$ \\
\hline $\begin{array}{l}\text { Lose your train of thought e.g. taking in information that sparks an idea, only } \\
\text { to take in more information and lose the first idea }\end{array}$ & $1 \square 2 \square 3 \square 4 \square 5 \square$ \\
\hline
\end{tabular}

Final Questions

\begin{tabular}{|l|l|}
\hline $\begin{array}{l}\text { Do you find yourself searching for the same information repeatedly? (phone numbers, website } \\
\text { addresses, etc.) }\end{array}$ & Yes / No \\
\hline Can you remember details of an email sent to you over a month ago? & Yes / No \\
\hline Could you locate this email? & Yes / No \\
\hline $\begin{array}{l}\text { Can you remember details of a webpage (that you don't frequently visit) you visited over a } \\
\text { month ago? }\end{array}$ & Yes / No \\
\hline Would you be able to locate that website? & Yes / No \\
\hline $\begin{array}{l}\text { Can you remember details of a book / magazine or journal article that you read over a month } \\
\text { ago? }\end{array}$ & Yes / No \\
\hline
\end{tabular}


Would you be able to locate information in that article / book?

Yes / No 


\section{Memory Aids}

Do you use devices or techniques to support your memory?

Yes / No

\begin{tabular}{|c|c|c|}
\hline Which of the following do you make use of? & $\begin{array}{l}\text { Paper-Based } \\
\text { Shopping lists } \\
\text { To-do lists } \\
\text { Post it notes } \\
\text { Notepads } \\
\text { Address books } \\
\text { Diary / journal } \\
\text { Others } \\
1 \\
2 \\
3 . \\
4 . \\
5 \\
\text { Computer-Based } \\
\text { PDA } \\
\text { Web log } \\
\text { Web favourites / bookmarks } \\
\text { Email folders } \\
\text { OS search tool } \\
\text { Use email to remind you to complete a } \\
\text { task. } \\
\text { Others } \\
1 . \\
2 . \\
3 . \\
4 . \\
5 .\end{array}$ & $\begin{array}{l}\square \\
\square \\
\square \\
\square \\
\square \\
\square \\
\square \\
\square \\
\square \\
\square \\
\square \\
\square \\
\square \\
\square \\
\square \\
\square \\
\square \\
\square \\
\square \\
\square \\
\square \\
\square \\
\square \\
\square \\
\square \\
\square\end{array}$ \\
\hline
\end{tabular}

